\title{
SOLITON THEORY AND HANKEL OPERATORS
}

\author{
SERGEI GRUDSKY AND ALEXEI RYBKIN
}

\begin{abstract}
Soliton theory and the theory of Hankel (and Toeplitz) operators have stayed essentially hermetic to each other. This paper is concerned with linking together these two very active and extremely large theories. On the prototypical example of the Cauchy problem for the Korteweg-de Vries (KdV) equation we demonstrate the power of the language of Hankel operators in which symbols are conveniently represented in terms of the scattering data for the Schrodinger operator associated with the initial data for the KdV equation. This approach yields short-cuts to already known results as well as to a variety of new ones (e.g. wellposedness beyond standard assumptions on the initial data) which are achieved by employing some subtle results for Hankel operators.
\end{abstract}

\section{Preface}

The title reflecting the final results of the work should have been formulated as something like "On the Inverse Scattering Transform for the Korteweg-de Vries equation on the line with essentially arbitrary initial data decaying sufficiently rapidly on the right half line". However, that would have narrowed the number of potential readers down to a rather small group of specialists interested in the socalled step-like initial data, which was not our intention. Hoping to draw attention of a much larger community of mathematicians and theoretical physicists whose lexicon intersects with ours, we have risked compilation of the title merely from the names of two enormously large areas which we have the goal to connect.

The name soliton theory in the title of the paper underlines that our main results belong to this theory. The term Hankel operators, in turn, should suggest that the exposition is based upon the theory of Hankel operators. While having experienced a boom at the same time, these two theories have not shown much of interaction. It is our main goal to demonstrate that the language of Hankel/Toeplitz operators is very natural for soliton theory (completely integrable systems) with lots of potentials. We would also like to capture the attention of the Hankel/Toeplitz operator community who may find something new in the theoretical aspect, but above all get acquainted with applications of Hankel operators to the inverse scattering transform for integrable systems, which may have a stimulating influence on the development of Hankel and Toeplitz operators.

Date: October, 2013.

1991 Mathematics Subject Classification. 34B20, 37K15, 47B35.

Key words and phrases. KdV equation, Hankel operators.

The first author is supported by PROMEP (México) via "Proyecto de Redes", by CONACYT grant 180049, and by Federal program "Scientific and Scientific-Pedagogical Personnel of Innovative Russia for the years 2007-2013" (contract No 14.A18.21.0873) .

The second author is supported in part by the NSF under grant DMS 1009673. 
Our purpose determines the style of the paper: maximally self-contained exposition with recall of basic definitions and auxiliary results.

We concentrate solely on the Korteweg-de Vries (KdV) case but it should be quite clear to anyone familiar with the area that our approach by no means is restricted to this case. Moreover, we believe that the interplay between soliton theory and Hankel operators may be even more interesting and fruitful for some other integrable systems with richer than KdV structures.

\section{INTRODUCTION}

Soliton theory originated in the mid 60s from the fundamental Gardner-GreeneKruskal-Miura discovery of what we now call the inverse scattering transform (IST) for the $\mathrm{KdV}$ equation. It is regarded as a major achievement of the 20s century connecting different branches of pure mathematics and theoretical physics with numerous applications ranging from hydrodynamics and nonlinear optics to astrophysics and elementary particle theory (see, e.g. the classical books [1, [61]). Conceptually, the IST is similar to the Fourier transform. In the context of the Cauchy problem for the KdV equation on the full line

$$
\begin{gathered}
\partial_{t} q-6 q \partial_{x} q+\partial_{x}^{3} q=0, \\
q(x, 0)=q(x),
\end{gathered}
$$

the IST method consists, as the standard Fourier transform method, of three steps:

Step 1. (direct transform)

$$
q(x) \longrightarrow S_{q},
$$

where $S_{q}$ is a new set of variables which turns (2.1) into a simple first order linear ODE for $S_{q}(t)$ with the initial condition $S_{q}(0)=S_{q}$.

Step 2. (time evolution)

Step 3. (inverse transform)

$$
S_{q} \longrightarrow S_{q}(t)
$$

$$
S_{q}(t) \longrightarrow q(x, t) \text {. }
$$

Similar methods have also been developed for many other evolution nonlinear PDEs, which are referred to as completely integrable 1 Each of steps 1-3 involves solving a linear equation that allows us to analyze integrable systems at the level unreachable by neither direct numerical methods nor standard PDE techniques. The study of a large variety of realizations of these steps for different integrable systems and initial conditions (including the analysis of the information that the IST yields about these systems) constitutes the core of soliton theory.

In the classical IST for (2.1)-(2.2), when $q$ is rapidly decaying as $|x| \rightarrow \infty$ (the socalled short range), $S_{q}$ is a set of scattering data associated with the Schrödinger operator $\mathbb{L}_{q}=-\partial_{x}^{2}+q$. By solving the Schrödinger equation $\mathbb{L}_{q} u=k^{2} u$ one finds $S_{q}=\left\{R(k),\left(\kappa_{n}, c_{n}\right)\right\}$, where $R(k), k \in \mathbb{R}$, is the reflection coefficient and $\left(\kappa_{n}, c_{n}\right), n=1,2, . ., N$, are so-called bound state data associated with with eigenvalues $-\kappa_{n}^{2}$ of $\mathbb{L}_{q}$. Step 2 readily yields

$$
S_{q}(t)=\left\{R(k) \exp \left(8 i k^{3} t\right), \kappa_{n}, c_{n} \exp \left(8 \kappa_{n}^{3} t\right)\right\} .
$$

\footnotetext{
${ }^{1}$ There is no precise meaning of complete integrability but the question "What is integrability?" has drawn much attention (see e.g. the survey 45).
} 
Step 3 amounts to solving the inverse scattering problem of recovering the potential $q(x, t)$ (which now depends on $t \geq 0$ ) from $S_{q}(t)$. This procedure comes with an explicit formula, called determinant or Dyson,

$$
q(x, t)=-2 \partial_{x}^{2} \log \operatorname{det}(I+\mathbb{H}(x, t)),
$$

where $\mathbb{H}(x, t)$ is the Hankel operator $\mathbb{H}\left(\varphi_{x, t}\right)$ with symbol

$$
\varphi_{x, t}(k)=R(k) \xi_{x, t}(k)+\sum_{n=1}^{N} \frac{c_{n} \xi_{x, t}\left(i \kappa_{n}\right)}{\kappa_{n}+i k} .
$$

Here $\xi_{x, t}(k)=\exp \left\{i\left(8 k^{3} t+2 k x\right)\right\}$ solely carries the dependence on $(x, t)$. This puts us in the context of the theory of Hankel operators and Steps 1-3 can now be combined to read

$$
q(x) \longrightarrow \mathbb{H}\left(\varphi_{x, t}\right) \longrightarrow q(x, t) .
$$

Note that there are many other methods to carry out Step 3. Historically, the first one, called Gelfand-Levitan-Marchenko, amounts to working with $\mathbb{H}(x, t)$ in the form of an integral (Marchenko) operator which kernel is the Fourier transform of $\varphi_{x, t}$. The most contemporary one is based upon the Riemann-Hilbert problem which is solved using techniques of singular integral equations. Overall, while both look quite similar, the latter is arguably much more powerful as the Fourier transform in a sense smears the dependence on $(x, t)$. Our approach also starts out from a Riemann-Hilbert problem (the basic scattering relation (6.8) ) which we solve in terms of Hankel operators in the form (4.4), but not in the Marchenko form. This gives us a direct access to the well-developed theory of Hankel operators where, in fact, the integral form is not used much either.

Thus (2.6) suggests the relevance of soliton theory to Hankel operators which theory is also extremely large. While both theories have been developed at about same time, there has been very little interaction between the two. The connection we have discussed so far is rather skin deep and does not offer immediate benefits to either theory. This is likely true if we stay within the realm of short range initial data $q$ but our goal is to go far beyond this.

Let us put our goal into historic context. An analog of the IST for periodic $q$ 's was found in 62. It rallies on the Floquet theory for $\mathbb{L}_{q}$ and analysis of Riemann surfaces and hence is much more complex than the short range case2. However, as emphasized in [49], (2.1)-(2.2) is completely integrable essentially only in these two cases. In fact, the question whether any well-posed problem (initial value, boundary value, etc.) for (2.1) ${ }^{3}$ could be solved by a suitable IST, has been raised in one form or another by many (see e.g. [1, 449, [59]) and some regard it as a major unsolved problem. Once we are outside of the scattering or periodic situations many real complications arise. The main question is of course what $S_{q}$ should be. A large amount of effort has been put into developing the IST on intervals (see, e.g., the recent sequel [27], [53], [54]). We only mention that $S_{q}$ consists of the certain spectral functions (some of which depend on the initial data and some on the boundary data) related through a nonlinear algebraric equation (the global relation 4 ).

\footnotetext{
${ }^{2}$ Unification of these two cases is offered in 1] as an open problem.

${ }^{3}$ Or any other integrable system.

${ }^{4}$ Solving this relation is the only nonlinear step in the IST procedure.
} 
Here we are interested in the initial value problem on the whole line but with $q$ outside of classes of short range or periodic functions. Much of known (rigorous) results are on some sort of "hybrids" of these two cases. Namely, physically important cases of short-range perturbations of a step function 5 (see e.g. [14, 23, [44, [50, 78, ) and two crystals fused together6 (24], 25]). Steps 1-3 are much more complicated than in the short range case and require subtle analysis which has been completed only recently. Certain cases of slowly decaying profiles have also received considerable attention (see e.g. [32], [56, [58]), but this situation is much less understood.

In the present paper we deal with initial data subject to

Hypothesis 2.1. Let $q$ be a real locally integrable function subject to

(1) (boundedness from below)

$$
\inf \operatorname{Spec}\left(\mathbb{L}_{q}\right)=-h_{0}^{2}>-\infty ;
$$

(2) (decay at $+\infty$ ) For some positive weight function $w(x) \geq x$

$$
\int^{\infty} w(x)|q(x)| d x<\infty .
$$

We show that under Hypothesis 2.17 (2.1)-(2.2) is globally well-posed and completely integrable in the sense that (2.6) can be explicitly realized. Note that our class of initial profiles, which we call step-like, is extremely large. The condition

$$
\operatorname{Sup}_{x} \int_{x}^{x+1} \max (-q, 0)<\infty
$$

is sufficient for (2.7) and is also necessary if $q \leq 0$. Therefore, any $q$ subject to Hypothesis 2.1 is essentially bounded from below, decay sufficiently fast at $+\infty$ but arbitrary otherwise.

The main feature of our situation is that we can do one sided scattering theory and define a suitable (right) reflection coefficient $R(k)$. The problem is that $R$ need not have smoothness and decay properties that the machinery of the classical IST relies on. We overcome these issues by extracting a part $A$ from $R$ (Propositions 7.12 and (7.10) which mimics the irregular behavior of $R(k)$ for real $k$ but admits an analytic continuation into the upper half plane. The rest of $R(k)$ is (in a sense) small and easily controlled by the behavior of $q$ at $+\infty$. What actually makes this split work is that $A$ can be chosen to keep all the necessary information about the negative spectrum of the whole operator $\mathbb{L}_{q}$. This split (written in a different form) was a crucial ingredient of our [68] in dealing with similar to $\mathrm{Hy}-$ pothesis 2.1 conditions but under extra hard-to-verify assumptions. The problem is that Hypothesis 2.1 does not rule out the case $|R(k)|=1$ for a.e. real $k$ which further complicates the situation. In the quantum mechanical sense, such $q$ 's are completely nontransparent for plane waves coming from $+\infty$. Examples include (1) functions growing (arbitrarily fast) at $-\infty$ (not quite physical), (2) Gaussian white noise on a left half line (like the stock market), (3) certain sparse sequences of bumps (Pearson blocks [65]), and (4) certain (random) slowly decaying at $x \rightarrow-\infty$

\footnotetext{
${ }^{5} \mathrm{~A}$ bore wave type initial profile.

${ }^{6}$ Soliton propogation on a periodic backgound.

${ }^{7}$ In fact, we will be a bit conservative regarding $w$ for some inessential reasons.

${ }^{8}$ I.e., $\left(\kappa_{n}, c_{n}\right)$ in the classical case.
} 
functions (Kotani potentials [51]), to mention just four. Note that if $R(k)$ is unimodular then all previously known approaches to step-like initial conditions break down in a serious way. Our approach developed in 69] could handle this case but some inconvenient conditions had to be imposed to rule out the possibility for $\mathbb{H}\left(\varphi_{x, t}\right)$ to have eigenvalue -1 for some $(x, t)$. Otherwise, as it follows from (2.4), $q(x, t)$ will develop a double pole type singularity at that point causing (2.1)-(2.2) to be ill-posed in many respects. One of our main contributions is showing that under Hypothesis 2.1 -1 never belongs to the spectrum of $\mathbb{H}\left(\varphi_{x, t}\right)$ and we prove it by means of Hankel/Toeplitz operators. It is curious to note that the analyticity of $\xi_{x, t}(k)=\exp \left\{i\left(8 k^{3} t+2 k x\right)\right\}$ is not essential here but rather its membership in the Sarason algebra $H^{\infty}+C$ (see Section 5 for definition), a fundamental object of the theory of Hankel/Toeplitz operators (see, e.g. [10, [46], 66]). The latter is established in 20] in a totally different context. It is this nontrivial fact that opens an access to the powerful machinery of Hankel/Toeplitz operators.

We also crucially use the Adamyan-Arov-Krein theory (see, e.g. [46], 66]) which provides us with a beautiful way to compute singular numbers of Hankel operators that, in turn, gives very accurate error estimates for KdV solutions. The analyticity of $\xi_{x, t}(k)$, not its membership in the Sarason algebra, becomes important here.

It is the use of the Sarason algebra and Adamyan-Arov-Krein theory that has let us conclude our study [68, 69] of complete integrability of (2.1)-(2.2) with step-like initial data. Moreover we see that many other results and problems of soliton theory become more transparent once translated into the language of Hankel operators. We are now convinced that the theory of Hankel/Toeplitz operators has potentially much more to offer to soliton theory encouraging closer interaction between two theories. This point of view is also reinforced by the recent papers [30, 31] where a totally different set of results on soliton theory was obtained using Hankel operators.

Our goal was to make the paper as self-contained and rigorous as possible supplemented with proofs of known results whenever it is instructive and can be done in a nice way. This always presents a challenge if you also want to keep the volume reasonable. We are not sure if our goal is achieved but we tried our best.

The paper is organized as follows. In Section 3 we merely list some of our notation and conventions and give a short review of Hardy and Gevrey classes. Sections 4 and 5 are devoted to reviewing some background information and preparing some facts about Hankel operators needed in the following sections. In Section [6] we recall some basics of the classical IST and reformulate it in terms of Hankel operators. In Section 7 we study the analytic structure of the reflection coefficient which is crucially used in the following sections. In Section 8 we state and prove some principal properties of our specific Hankel operator. In Section 9 we use the Adamyan-Arov-Krein classical theory to obtain subtle relations between the decay of singular numbers of our Hankel operator and properties of the initial data $q$. Section 10 is devoted to our main result, Theorem 10.2. Its corollaries, as well as related discussions and historical comments are given in Section 11. In the final Section 12 we state some open problems.

\section{Notation and Function Classes}

3.1. Basic notation and conventions. We follow standard notation accepted in Analysis. For number sets: $\mathbb{N}_{0}=\{0,1,2, \ldots\}, \mathbb{R}$ is the real line, $\mathbb{R}_{ \pm}=(0, \pm \infty), \mathbb{C}$ is the complex plane, $\mathbb{C}^{ \pm}=\{z \in \mathbb{C}: \pm \operatorname{Im} z>0\} . \bar{z}$ is the complex conjugate of $z$. 
Besides number sets, black board bold letters will also be used for (linear) operators. In particular, $\mathbb{I}$ denotes the identity operator. $\mathbb{A}^{*}$ stands for the adjoint of a linear operator $\mathbb{A}$ on a Hilbert space. For a given compact operator $\mathbb{A}$ on a Hilbert space, we recall that its $n$-th singular value $s_{n}(\mathbb{A})$ is defined as the $n$-th eigenvalue of the operator $\left(\mathbb{A}^{*} \mathbb{A}\right)^{1 / 2}$. We say that $\mathbb{A}$ is in the Shatten-von Neumann class $\mathfrak{S}_{p}, 0<p \leq \infty$, if $\left\{s_{n}(\mathbb{A})\right\} \in l^{p}$. (Here $l^{p}$ stands for the space of all sequences $\left(x_{n}\right)$ such that $\left.\Sigma_{n}\left|x_{n}\right|^{p}<\infty\right)$. We write $\mathbb{A} \geq 0$ if $\langle\mathbb{A} f, f\rangle \geq 0$ for any $f$ from the domain of $\mathbb{A}$. $(\langle\cdot, \cdot\rangle$ stands for the inner product). $\mathbb{A}>0$ means that $\mathbb{A} \geq 0$ and $\langle\mathbb{A} f, f\rangle=0$ iff $f=0$. We write $\mathbb{A} \geq \mathbb{B}$ if $\mathbb{A}-\mathbb{B} \geq 0$.

Some other notation: $\chi(x)$ is the Heaviside function, $\chi_{c}(x):=\chi(x-c)$, and

$$
\xi_{x, t}(k):=\exp \left\{i\left(8 k^{3} t+2 k x\right)\right\}, \quad \xi_{x}(k):=\xi_{x, 0}(k)=\exp \{2 i k x\},
$$

is a fundamental to the IST function.

We frequently (but not always) arrange the variables of a function in the order of their importance. E.g. $f(x, p)$ should suggest that $x$ is the main variable and $p$ is a parameter. To reduce the amount of clutter we will often drop variables of functions whenever it causes no confusion, abbreviate $\int f(x) d x=\int f$ and, when appropriate, write $y \lesssim_{a} x$ in place of $y \leq C_{a} x$ with some $C_{a}>0$ dependent on $a$ but independent of $x$. If $C$ is a universal constant we then write $y \lesssim x$.

We use $\rightrightarrows$ to denote uniform convergence. In particular, we agree to write $f_{n}(z) \rightrightarrows f(z)$ in $\mathbb{C}^{+}$if $f_{n}(z)$ converges to $f(z)$ uniformly on compact subsets of $\mathbb{C}^{+}$containing no singularities of $f_{n}(z), f(z)$.

We have the following agreement on \pm statements: $P_{ \pm} \Rightarrow Q_{ \pm}$means two separate statements $P_{+} \Rightarrow Q_{+}, P_{-} \Rightarrow Q_{-}$. We then use $P_{ \pm}$as a single noun. If it is used as a plural noun then $P_{ \pm}$means $P_{+}$and $P_{-}$. Since quantities labeled with + will appear more frequently whenever convenient we also drop + and just write $P$ for $P_{+}$. (But we will never suppress the subscript -.)

3.2. Basic function classes. As usual, $L^{p}(S), 0<p \leq \infty$, is the Lebesgue space on a set $S$. Typically $S=\mathbb{R}$ which justifies the abbreviation

$$
\int \stackrel{\text { def }}{=} \int_{\mathbb{R}}, \quad L^{p} \stackrel{\text { def }}{=} L^{p}(\mathbb{R}) \text {. }
$$

And $\left(\partial_{x}^{n}:=\partial^{n} / \partial x^{n}, n \in \mathbb{N}_{0}\right)$

$$
\begin{aligned}
& C \stackrel{\text { def }}{=}\left\{f: f \text { is continuous on } \mathbb{R}, \lim _{x \rightarrow \infty} f(x)=\lim _{x \rightarrow-\infty} f(x) \neq \pm \infty\right\}, \\
& C^{n} \stackrel{\text { def }}{=}\left\{f: \partial_{x}^{n} f \in C\right\}, n \in \mathbb{N}_{0} ; C^{\infty} \stackrel{\text { def }}{=} \cap_{n \in \mathbb{N}_{0}} C^{n} .
\end{aligned}
$$

3.3. Hardy classes. To translate our problem into the language of Hankel/Toeplitz operators some common definitions and facts are in order [29].

A function $f$ analytic in $\mathbb{C}^{ \pm}$is in the Hardy space $H_{ \pm}^{p}$ for some $0<p \leq \infty$ if

$$
\|f\|_{H_{ \pm}^{p}}^{p} \stackrel{\text { def }}{=} \sup _{y>0}\|f(\cdot \pm i y)\|_{p}<\infty .
$$

We remind that by our convention we set $H^{p}=H_{+}^{p}$.

It is a fundamental fact of the theory of Hardy spaces that any $f(z) \in H_{ \pm}^{p}$ with $0<p \leq \infty$ has non-tangential boundary values $f(x \pm i 0)$ for almost every (a.e.) $x \in \mathbb{R}$ and

$$
\|f\|_{H_{ \pm}^{p}}=\|f(\cdot \pm i 0)\|_{L^{p}} \stackrel{\text { def }}{=}\|f\|_{p} .
$$


Classes $H_{ \pm}^{\infty}$ and $H_{ \pm}^{2}$ will be particularly important. $H_{ \pm}^{\infty}$ is the algebra of uniformly bounded in $\mathbb{C}^{ \pm}$functions and $H_{ \pm}^{2}$ is the Hilbert space with the inner product induced from $L^{2}$ :

$$
\langle f, g\rangle_{H_{ \pm}^{2}}=\langle f, g\rangle_{L^{2}}=\langle f, g\rangle=\int f \bar{g} .
$$

It is well-known that $L^{2}=H^{2} \oplus H_{-}^{2}$, the orthogonal (Riesz) projection $\mathbb{P}_{ \pm}$onto $H_{ \pm}^{2}$ being given by

$$
\left(\mathbb{P}_{ \pm} f\right)(x)= \pm \frac{1}{2 \pi i} \lim _{\varepsilon \rightarrow 0+} \int \frac{f(s) d s}{s-(x \pm i \varepsilon)} \stackrel{\text { def }}{=} \pm \frac{1}{2 \pi i} \int \frac{f(s) d s}{s-(x \pm i 0)} .
$$

Of course

$$
\mathbb{P}_{ \pm}^{*}=\mathbb{P}_{ \pm}, \mathbb{P}_{ \pm}^{2}=\mathbb{P}_{ \pm}, \mathbb{P}_{+}+\mathbb{P}_{-}=\mathbb{I}
$$

Notice that for any $f \in H^{2}$ and $\lambda \in \mathbb{C}^{+}$

$$
\mathbb{P}_{-} \frac{f(\cdot)}{\cdot-\lambda}=\mathbb{P}_{-} \frac{f(\cdot)-f(\lambda)}{\cdot-\lambda}+\mathbb{P}_{-} \frac{f(\lambda)}{\cdot-\lambda}=\frac{f(\lambda)}{\cdot-\lambda} .
$$

The operators given by (3.3) remain bounded from $L^{p}$ to $H_{ \pm}^{p}$ for $1<p<\infty$. For $L^{\infty}$ the (regularized) Riesz projection

$$
\begin{aligned}
\left(\widetilde{\mathbb{P}}_{ \pm} f\right)(x) & =(x+i)\left(\mathbb{P}_{ \pm} \frac{1}{\cdot+i} f\right)(x), \\
& = \pm \frac{1}{2 \pi i} \int\left(\frac{1}{s-(x \pm i 0)}-\frac{1}{s+i}\right) f(s) d s, \quad f \in L^{\infty},
\end{aligned}
$$

is clearly well-defined and $\widetilde{\mathbb{P}}_{ \pm} f$ is analytic in $\mathbb{C}^{ \pm}$. Moreover $\widetilde{\mathbb{P}}_{ \pm}$is bounded from $L^{\infty}$ to $\operatorname{BMOA}\left(\mathbb{C}^{ \pm}\right)$. I.e.

$$
f \in L^{\infty} \Longrightarrow \widetilde{\mathbb{P}}_{ \pm} f \in \operatorname{BMOA}\left(\mathbb{C}^{ \pm}\right) .
$$

Here BMOA $\left(\mathbb{C}^{ \pm}\right)$stands for the well-known class of analytic in $\mathbb{C}^{ \pm}$functions from $\mathrm{BMO}(\mathbb{R})$. The space $\mathrm{BMO}(\mathbb{R})$ (Bounded Mean Oscillation) consists of locally integrable functions $f$ on $\mathbb{R}$ satisfying ( $I$ is a bounded interval)

$$
\|f\|_{\mathrm{BMO}}=\sup _{I \in \mathbb{R}} \frac{1}{|I|} \int_{I}\left|f-f_{I}\right|<\infty, \quad f_{I} \stackrel{\text { def }}{=} \frac{1}{|I|} \int_{I} f .
$$

It is important to us that

$$
\widetilde{\mathbb{P}}_{+} f+\widetilde{\mathbb{P}}_{-} f=f, f \in L^{\infty},
$$

which immediately follows from (3.4), (3.6), and (3.7). One can also see from (3.7) that if $f \in L^{2}$ then $\widetilde{\mathbb{P}}_{+} f=\mathbb{P}_{+} f+$ const, and $\widetilde{\mathbb{P}}_{+} f=\mathbb{P}_{+} f=f$ if $f \in H^{2}$.

We will occasionally use Blaschke products

$$
B(z)=\prod_{n \geq 1} b_{n}(z), b_{n}(z)=\frac{1+z_{n}^{2}}{\left|1+z_{n}^{2}\right|} \frac{z-z_{n}}{z-\overline{z_{n}}} .
$$

Recall that the product 19 in (3.10) is uniformly convergent on compact sets in $\mathbb{C}^{+}$ iff the Blaschke condition

$$
\sum_{n \geq 1} \frac{\operatorname{Im} z_{n}}{1+\left|z_{n}\right|^{2}}<\infty
$$

\footnotetext{
${ }^{9}$ Assuming that $i$ is not a zero of $B$. If $i$ is a zero then (3.10) should be modified accordingly.
} 
is satisfied. Moreover $B \in H^{\infty},|B(z)| \leq 1$ if $z \in \mathbb{C}^{+}, B$ is unimodular a.e. on $\mathbb{R}$, and has zeros in $\mathbb{C}^{+}$at $\left\{z_{n}\right\}$.

Most of our Blaschke products will have purely imaginary zeros $\left\{i x_{n}\right\}$ of multiplicity 1. In this case (3.11) is equivalent to $\left\{x_{n}\right\} \in l^{1}$. Observe that such $B$ is continuous on the real line away from 0 . The following simple statement is a product analog of the Weierstrass M-test.

Proposition 3.1. Let $\left\{x_{n}(p)\right\}$ be a positive sequence dependent on a parameter $p$ (say positive). Suppose that $x_{n}(p) \leq \lim _{p \rightarrow \infty} x_{n}(p)=: x_{n}<\infty$ for each $n$. If $\left\{x_{n}\right\} \in l^{1}$ then

$$
B_{p}(z):=\prod_{n \geq 1} \frac{z-i x_{n}(p)}{z+i x_{n}(p)} \underset{p \rightarrow \infty}{\rightrightarrows} B(z)=\prod_{n \geq 1} \frac{z-i x_{n}}{z+i x_{n}} \text { in } \mathbb{C}^{+} .
$$

Proof. Setting $b_{n}^{p}(z):=\frac{z-i x_{n}(p)}{z+i x_{n}(p)}, b_{n}(z):=\frac{z-i x_{n}}{z+i x_{n}}$ we have for each $z$ away from $\left\{i x_{n}\right\}$

$$
\left|B_{p}-B\right|=|B|\left|\prod_{n \geq 1} b_{n}^{p} / b_{n}-1\right| \leq\left|\prod_{n \geq 1} b_{n}^{p} / b_{n}-1\right| .
$$

We are done if we show that on each compact set $K$ in $\mathbb{C}^{+} \backslash \cup_{p>0}\left\{i x_{n}(p)\right\}$

$$
\begin{aligned}
f_{p}(z) & :=\log \prod_{n \geq 1} b_{n}^{p}(z) / b_{n}(x) \\
& =\sum_{n \geq 1} \log \left(1+\frac{i\left(x_{n}-x_{n}(p)\right)}{z-i x_{n}}\right)+\sum_{n \geq 1} \log \left(1+\frac{i\left(x_{n}-x_{n}(p)\right)}{z+i x_{n}(p)}\right) \\
& \rightrightarrows 0, \quad p \rightarrow \infty .
\end{aligned}
$$

But uniformly on $K$

$$
\begin{aligned}
\left|f_{p}(z)\right| & \lesssim \sum_{n \geq 1}\left(\left|z-i x_{n}\right|^{-1}+\left|z+i x_{n}(p)\right|^{-1}\right)\left(x_{n}-x_{n}(p)\right) \\
& \lesssim_{K} \sum_{n \geq 1}\left(x_{n}-x_{n}(p)\right)
\end{aligned}
$$

and the standard Weierstrass M-test applies.

Finally, a set $S \subset \mathbb{C}^{+}$is called a uniqueness set for $H^{2}$ if for any $f \in H^{2}$

$$
\left.f\right|_{S}=0 \Longrightarrow f=0 \text {. }
$$

Otherwise $S$ is called a nonuniqueness set for $H^{2}$. Obviously, a nonuniqueness set must satisfy the Blaschke condition. Thus, if a set $S$ is uncountable then it is a uniqueness set and if $S$ is countable then it must fail the Blaschke condition.

3.4. Gevrey classes. We will need the Gevrey classes $G^{\alpha}, \alpha \geq 1$, of smooth functions $f$ such that

$$
\left|\partial_{x}^{n} f(x)\right| \lesssim_{f} Q_{f}^{n}(n !)^{\alpha} \text { for all } x \text { and } n,
$$

with some $Q_{f}>0$. Note that $G^{1}$ is the set of real analytic function. Following [21] we call $F(x, y)$ a pseudoanalytic extension of $f(x)$ to $\mathbb{C}$ if

$$
F(x, 0)=f(x) \text { and } \bar{\partial} F(x, y) \rightarrow 0, y \rightarrow 0,
$$


where $\bar{\partial} \stackrel{\text { def }}{=}(1 / 2)\left(\partial_{x}+i \partial_{y}\right)$ The statement $f \in G^{\alpha}$ is equivalent 21 to the statement that $f$ admits a pseudoanalytic extension $F$ such that for some $Q>0$

$$
|\bar{\partial} F(x, y)| \lesssim_{f} \exp \left\{-Q|y|^{-\frac{1}{\alpha-1}}\right\} .
$$

\section{Hankel Operators, Basic Definitions}

A Hankel operator is an infinitely dimensional analog of a Hankel matrix, a matrix whose $(j, k)$ entry depends only on $j+k$. I.e. a matrix $\Gamma$ of the form

$$
\Gamma=\left(\begin{array}{cccc}
\gamma_{1} & \gamma_{2} & \gamma_{3} & \ldots \\
\gamma_{2} & \gamma_{3} & \cdots & \\
\gamma_{3} & \cdots & & \\
\cdots & & & \gamma_{n}
\end{array}\right) .
$$

Definitions (and properties) of Hankel operators depend on specific spaces and need not be equivalent. We consider Hankel operators on $H^{2}$ (c.f. [46], 66]).

Let

$$
(\mathbb{J} f)(x) \stackrel{\text { def }}{=} f(-x)
$$

be the operator of reflection on $L^{2}$. It is clearly an isometry with the obvious properties

$$
\begin{aligned}
\mathbb{J}^{*} & =\mathbb{J}, \mathbb{J}^{2}=\mathbb{I}, \mathbb{J}^{-1}=\mathbb{J} . \\
\mathbb{J}(\varphi f) & =(\mathbb{J} \varphi) \mathbb{J} f, \varphi \in L^{\infty}, f \in L^{2} \\
\mathbb{J P}_{\mp} & =\mathbb{P}_{ \pm} \mathbb{J} .
\end{aligned}
$$

Definition 4.1 (Hankel and Toeplitz operators). Let $\varphi \in L^{\infty}$. The operators $\mathbb{H}(\varphi)$ and $\mathbb{T}(\varphi)$ defined by

$$
\mathbb{H}(\varphi) f=\mathbb{J} \mathbb{P}_{-} \varphi f, \text { and } \mathbb{T}(\varphi) f=\mathbb{P}_{+} \varphi f, \quad f \in H^{2},
$$

are called respectively the Hankel and Toeplitz operators with the symbol $\varphi$.

Due to (4.3), both $\mathbb{H}(\varphi)$ and $\mathbb{T}(\varphi)$ act from $H^{2}$ to $H^{2}$. Note that while $\mathbb{H}(\varphi)$ and $\mathbb{T}(\varphi)$ look alike, they are different parts of the multiplication operator

$$
\varphi f=\mathbb{J} \mathbb{H}(\varphi) f+\mathbb{T}(\varphi) f, \quad f \in H^{2},
$$

and therefore are quite different. The Toeplitz operator will play only an auxiliary role in our consideration.

Directly from the definition, $\|\mathbb{H}(\varphi)\| \leq\|\varphi\|_{\infty}$ but much more subtle statements will be required.

Theorem 4.2 (Widom, 1960). Let $\varphi$ be unimodular. Then $\|\mathbb{H}(\varphi)\|<1$ iff $\mathbb{T}(\varphi)$ is left invertible.

The sufficiency in this theorem is a direct consequence of (4.5) but the necessity is a really deep result (see [10, sect. 2.20).

We will occasionally have to deal with certain unbounded symbols (more exactly, from $\mathrm{BMO}$ ) which nevertheless produce bounded Hankel operators. In such cases we define $\mathbb{H}(\varphi)$ first on the set

$$
\mathfrak{H}_{2} \stackrel{\text { def }}{=}\left\{f \in H^{2}: f \in C^{\infty}, f(z)=o\left(z^{-2}\right), z \rightarrow \infty, \operatorname{Im} z \geq 0\right\},
$$


dense [29] in $H^{2}$ by

$$
\mathbb{H}(\varphi) f=\mathbb{P} \mathbb{P}_{-} \varphi f, \quad f \in \mathfrak{H}_{2},
$$

and then extend (4.7) to the whole $H^{2}$ retaining the same notation $\mathbb{H}(\varphi)$ for the extension.

Since obviously $\mathbb{H}(\varphi+h)=\mathbb{H}(\varphi)$, for any $h \in H^{\infty}$, only the part of $\varphi$ analytic in $\mathbb{C}^{-}$is essential. We call (see (3.6))

$$
\Phi \stackrel{\text { def }}{=} \widetilde{\mathbb{P}}_{-\varphi}
$$

the principal (co-analytic) part of the symbol $\varphi$. It need not be in $L^{\infty}$ but due to (3.8) $\Phi \in \operatorname{BMOA}\left(\mathbb{C}^{-}\right)$. The next statement allows us to define Hankel operators with such symbols.

Theorem 4.3. Let $\varphi \in L^{\infty}$ and $h \in B M O A\left(\mathbb{C}^{+}\right)$. Then $\mathbb{H}(\varphi+h)$ is well-defined, bounded and

$$
\mathbb{H}(\varphi+h)=\mathbb{H}(\varphi)
$$

Consequently

$$
\mathbb{H}(\varphi)=\mathbb{H}(\Phi) .
$$

Proof. As well-known [29] every BMOA function $h$ is subject to $h(x) /\left(1+x^{2}\right) \in$ $L^{1}$ and one can easily see that $h f \in H^{2}$ if $f \in \mathfrak{H}_{2}$. Hence $\mathbb{P}_{-} h f=0$ and (4.9) holds on the set $\mathfrak{H}_{2}$. Therefore (4.9) can be closed to the whole $H^{2}$ and $\mathbb{H}(\varphi+h)$ is well-defined in the sense discussed above, bounded and (4.9) holds. By (3.9) $\varphi=\widetilde{\mathbb{P}}_{-} \varphi+\widetilde{\mathbb{P}}_{+} \varphi$ and (4.10) follows from (4.9) with $h=-\widetilde{\mathbb{P}}_{+} \varphi$ which, by (3.8), is in $\operatorname{BMOA}\left(\mathbb{C}^{+}\right)$.

If $\varphi \in \mathrm{BMO}$, then there exist $\varphi_{1}, \varphi_{2} \in L^{\infty}$ such that

$$
\varphi=\varphi_{1}+\widetilde{\mathbb{P}} \varphi_{2}
$$

and Theorem 4.3 immediately implies

Proposition 4.4. Let $\varphi \in B M O$, then $\mathbb{H}(\varphi)$ is well-defined and bounded.

The final statement of this section trivially follows from (4.1)-(4.3).

Proposition 4.5. $\mathbb{H}(\varphi)$ is selfadjoint if $\mathbb{J} \varphi=\bar{\varphi} \%$

In the context of integral operators the Hankel operator is usually defined as an integral operator on $L^{2}\left(\mathbb{R}_{+}\right)$whose kernel depends on the sum of the arguments

$$
(\mathbb{H} f)(x)=\int_{0}^{\infty} h(x+y) f(y) d y, f \in L^{2}\left(\mathbb{R}_{+}\right), x \geq 0
$$

and it is this form that Hankel operators typically appear in the inverse scattering formalism. One can show that the Hankel operator $\mathbb{H}$ defined by (4.12) is unitary equivalent to $\mathbb{H}(\varphi)$ with the symbol $\varphi$ equal to the Fourier transform of $h$. We emphasize though that the form (4.12) does not prove to be convenient for our purposes and also $h$ is in general not a function but a distribution.

Finally, we also note that $\mathbb{H}(\varphi)$ is unitary equivalent to the operator $\chi \mathbb{F}^{-1} \varphi \mathbb{F}^{-1}$ on $L^{2}\left(\mathbb{R}_{+}\right)$. Here $\mathbb{F}$ is the Fourier transform. However our previous experience suggests that this realization of the Hankel operator has some technical disadvantages to (4.4). 


\section{HANKel Operators AND The SARASON ALGEBra $H^{\infty}+C$}

The set $H^{\infty}+C$ is one of the most common function classes in the theory of Hankel and Toeplitz operators. By definition

$$
H^{\infty}+C \stackrel{\text { def }}{=}\left\{f: f=h+g, h \in H^{\infty}, g \in C\right\} .
$$

Theorem 5.1 (Sarason, 1967). $H^{\infty}+C$ is a closed sub-algebra of $L^{\infty}$.

The importance of $H^{\infty}+C$ in the context of Hankel operators is due to the following fundamental theorem.

Theorem 5.2 (Hartman, 1958). Let $\varphi \in L^{\infty}$. Then $\mathbb{H}(\varphi)$ is compact iff $\varphi \in$ $H^{\infty}+C$. I.e. $\mathbb{H}(\varphi)$ is compact iff $\mathbb{H}(\varphi)=\mathbb{H}(g)$ with some $g \in C$.

For Hankel operators appearing in completely integrable systems the membership of the symbol in $H^{\infty}+C$ is far from being obvious. This may be part of the reason why the powerful machinery of Hankel operators has not made it to solution theory. The following statement will be crucial to our approach.

Theorem 5.3 (Grudsky, 2001). Let $p(x)$ be a real polynomial with a positive leading coefficient such that

$$
p(-x)=-p(x)
$$

Then

$$
e^{i p} \in H^{\infty}+C .
$$

Moreover, there exist an infinite Blaschke product $B$ and a unimodular function $u \in C$ such that

$$
e^{i p}=B u \text {. }
$$

It is worth mentioning that this theorem is a particular case of a more general statement originally obtained in 38 . (see also 20]) for the case of the unit circle and reformulated for the real line in [9] (see also recent 39]). This statement says that Theorem 5.3 holds not only for polynomial but any function $f$ such that

$$
\lim _{x \rightarrow \infty} \inf \frac{x f^{\prime \prime}(x)}{f^{\prime}(x)}>-2, \lim _{x \rightarrow \infty} \frac{x f^{\prime \prime}(x)}{f^{\prime}(x)^{2}}=0, \lim _{x \rightarrow \infty} \frac{\sqrt{x} f^{\prime \prime}(x)}{f^{\prime}(x)^{3 / 2}}=0 .
$$

We emphasize that functions of the form $e^{i p}$ commonly appear in the IST approach to completely integrable PDEs. For instance, in the KdV case

$$
p(\lambda)=t \lambda^{3}+x \lambda
$$

with real $x$ (spatial variable) and positive $t$ (time). Note that for polynomials $p$ of even order, Theorem 5.3 fails.

Definition 5.4. A function $f \in H^{\infty}+C$ is said invertible in $H^{\infty}+C$ if $1 / f \in$ $H^{\infty}+C$. Similarly, $f$ is not invertible in $H^{\infty}+C$ if $1 / f \notin H^{\infty}+C$.

This concept is very important in the connection with invertibility of Toeplitz operators, as the following theorem suggests (see, e.g. [10, 20]).

Theorem 5.5. Let $\varphi \in H^{\infty}+C$ and $1 / \varphi \in L^{\infty}$. Then

$$
\begin{aligned}
& 1 / \varphi \notin H^{\infty}+C \Longrightarrow \mathbb{T}(\varphi) \text { is left-invertible, } \\
& 1 / \varphi \in H^{\infty}+C \Longrightarrow \mathbb{T}(\varphi) \text { is Fredholm. }
\end{aligned}
$$


Lemma 5.6. Let $B$ be an infinite Blaschke product, $u \in H^{\infty}+C$ and unimodular. Then $\varphi=B u$ is not invertible in $H^{\infty}+C$.

Proof. (By contradiction). Since $B \in H^{\infty}$, due to the algebraic property (Theorem 5.1) of $H^{\infty}+C$, one has $\varphi \in H^{\infty}+C$. Assume that $\varphi$ is invertible in $H^{\infty}+C$, i.e. $1 / \varphi \in H^{\infty}+C$. Then by (5.5) $\mathbb{T}(\varphi)$ is Fredholm that forces $\mathbb{T}(B)$ to be Fredholm too. Indeed, $B \in H^{\infty}$ and, since $\varphi=B u$,

$$
1 / B=u \cdot 1 / \varphi \in H^{\infty}+C .
$$

Thus $B$ is invertible in $H^{\infty}+C$ and (5.5) holds. Hence $\mathbb{T}(\bar{B})=\mathbb{T}(1 / B)$ is also Fredholm and therefore by definition

$$
\operatorname{dim} \operatorname{ker} \mathbb{T}(\bar{B})<\infty .
$$

We now show that (5.6) may not hold for $B$ with infinitely many zeros $\left\{z_{k}\right\}$, which creates a desired contradiction. To this end consider the Blaschke product (3.10)

and set

$$
B(x)=\prod b_{n}(x), b_{n}=c_{n}\left(\frac{x-z_{n}}{x-\overline{z_{n}}}\right)
$$

Clearly $f_{n} \in H^{2}$ and

$$
f_{n}(x):=c_{n}\left(x-\overline{z_{n}}\right)^{-1} .
$$

$$
\mathbb{T}(\bar{B}) f_{n}=\mathbb{P}_{+} \bar{B} f_{n}=\mathbb{P}_{+} \overline{\overline{c_{n}}\left(\cdot-z_{n}\right)^{-1} B}=\mathbb{P}_{+}\left(\cdot-z_{n}\right)^{-1} \overline{B_{n}},
$$

where $B_{n}=B / b_{n}$. But $\overline{B_{n}} \in H_{-}^{\infty}$ and $\left(x-z_{n}\right)^{-1} \in H_{-}^{2}$. Hence

$$
\left(x-z_{n}\right)^{-1} \overline{B_{n}(x)} \in H_{-}^{2}
$$

and

$$
\mathbb{T}(\bar{B}) f_{n}=0 .
$$

Therefore $f_{n} \in \operatorname{ker} \mathbb{T}(\bar{B})$ and the lemma is proven as $\left\{f_{n}\right\}$ are linearly independent.

Note that Lemma 5.6 is entirely about $H^{\infty}+C$ but its proof, as often happens in this circle of issues, relies on operator theoretical arguments.

The next important claim directly follows from Theorem 5.3 and Lemma 5.6 .

Theorem 5.7. Let $u$ be a unimodular function from $H^{\infty}+C$ and $e^{i p}$ as in Theorem 5.3. Then $e^{i p} u$ is not invertible in $H^{\infty}+C$.

Combining Theorems 4.2 and 5.5 yields

Theorem 5.8. If $\varphi \in H^{\infty}+C$ and unimodular but not invertible then

$$
\|\mathbb{H}(\varphi)\|<1 \text {. }
$$

Proof. By Theorem [5.5, $\mathbb{T}(\varphi)$ is left-invertible. By Theorem 4.2 we have (5.7).

While an immediate consequence of Theorems 5.8 and 5.3 , the following theorem is vital to our approach.

Theorem 5.9. If $u \in H^{\infty}+C,|u|=1$, and $p$ is as in Theorem 5.3, then

$$
\left\|\mathbb{H}\left(e^{i p} u\right)\right\|<1 .
$$

Theorem 5.10. If $\varphi \in H^{\infty}+C$ is not unimodular but $\|\varphi\|_{\infty} \leq 1$ and $\mathbb{J} \varphi=\bar{\varphi}$ then (5.7) holds. 
Proof. (By contradiction) Assume that $\|\mathbb{H}(\varphi)\|=1$. Since $\mathbb{H}(\varphi)$ is selfadjoint and compact (by Proposition 4.5 and the Hartman theorem respectively), $\mathbb{H}(\varphi)$ has a unimodular eigenvalue $\lambda(\lambda= \pm 1)$. For the associated normalized eigenfunction $f \in H^{2}$ we have by (4.3)

$$
\langle\mathbb{H}(\varphi) f, f\rangle=\left\langle\varphi f, \mathbb{P}_{-} \mathbb{J} f\right\rangle=\langle\varphi f, \mathbb{J} f\rangle
$$

and hence by the Cauchy inequality

$$
\begin{aligned}
|\langle\mathbb{H}(\varphi) f, f\rangle|^{2} & \leq\left(\int|\varphi||f||\mathbb{J} f|\right)^{2} \leq \int|\varphi||f|^{2} \int|\varphi||\mathbb{J} f|^{2} \\
& \leq \int|\varphi||f|^{2} \int|\mathbb{J} f|^{2}=\int|\varphi||f|^{2} \\
& =\int_{S}|\varphi||f|^{2}+\int_{\mathbb{R} \backslash S}|\varphi||f|^{2}<\|f\|_{2}^{2}=1,
\end{aligned}
$$

where $S$ is a set of positive Lebesgue measure where $|\varphi|<1$ a.e. Here we have used the fact that $f \in H^{2}$ and hence cannot vanish on $S$. The inequality (5.8) implies that $|\lambda|<1$ which is a contradiction.

\section{The Classical IST and Hankel operators}

In this section we review some basics of the classical IST and prepare the necessary bulk of formulas (see, e.g. [19, [57]). We will also demonstrate the convenience of the Hankel operator approach to the Cauchy problem for the KdV equation in the classical situation of initial data decaying fast enough. Some derivations are given whenever we have a concise way to do so.

Through this section we assume that the initial profile $q$ in (2.1)-(2.2) is real and short range, i.e. $(1+|x|) q(x) \in L^{1}$. In the sequel we refer to such initial data as classical.

6.1. Direct scattering problem. Associate with $q$ the full line Schrödinger operator $\mathbb{L}_{q}=-\partial_{x}^{2}+q(x)$. As well-known, $\mathbb{L}_{q}$ is self-adjoint on $L^{2}$ and

$$
\operatorname{Spec}\left(\mathbb{L}_{q}\right)=\left\{-\kappa_{n}^{2}\right\}_{n=1}^{N} \cup \mathbb{R}_{+} .
$$

The singular spectrum of $\mathbb{L}_{q}$ consists of a finite number of simple negative eigenvalues $\left\{-\kappa_{n}^{2}\right\}$, called bound states, and absolutely continuous (a.c.) two fold component filling $\mathbb{R}_{+}$. There is no singular continuous spectrum. Two linearly independent (generalized) eigenfunctions of the a.c. spectrum $\psi_{ \pm}(x, k), k \in \mathbb{R}$, can be chosen to satisfy

$$
\psi_{ \pm}(x, k)=e^{ \pm i k x}+o(1), \partial_{x} \psi_{ \pm}(x, k) \mp i k \psi_{ \pm}(x, k)=o(1), \quad x \rightarrow \pm \infty .
$$

The functions $\psi_{ \pm}$are referred to as Jost solutions of the Schrödinger equation

$$
\mathbb{L}_{q} \psi=k^{2} \psi \text {. }
$$

We summarize the properties of $\psi_{ \pm}$in

Theorem 6.1 (On Jost solutions). The Jost solutions $\psi_{ \pm}(x, k)$ are analytic for $\operatorname{Im} k>0$ and continuous for $\operatorname{Im} k \geq 0$. Moreover as $k \rightarrow \infty, \operatorname{Im} k \geq 0$,

$$
\psi_{ \pm}(x, k)=e^{ \pm i k x}\left(1 \pm \frac{i}{2 k} \int_{x}^{ \pm \infty} q+O\left(\frac{1}{k^{2}}\right)\right)
$$


and

$$
\psi_{ \pm}(x,-k)=\overline{\psi_{ \pm}(x, k)}, k \in \mathbb{R} .
$$

This theorem is nearly folklore. One rewrites (6.2) as $\psi^{\prime \prime}+k^{2} \psi=q(x) \psi$ and then solves it by variation of parameters (keeping $q \psi$ as the non homogeneous term) with boundary conditions (6.1). The integral equation for $\psi_{ \pm}$obtained this way is Volterra-type and thus the (necessarily convergent) Neumann series obtained by iteration readily yields the conclusions of Theorem 6.1 .

To remove the oscillatory behavior of $\psi_{ \pm}$let us introduce the functions, sometimes called Faddeev,

$$
y_{ \pm}(k, x):=e^{\mp i k x} \psi_{ \pm}(x, k) .
$$

The function $y:=y_{+}$will be used more frequently. Its properties [19] are given in

Theorem 6.2 (On Faddeev functions). For any $x$, the function $y(k, x)$ is analytic in $\mathbb{C}^{+}$, continuous on the real line and

$$
y(k, x) \rightarrow 1,|k| \rightarrow \infty, \operatorname{Im} k \geq 0 .
$$

All zeros of $y(\cdot, x)$ in $\mathbb{C}^{+}$are imaginary and for their number $N_{x}$ we have

$$
N_{x} \leq \int_{x}^{\infty}(s-x)|q(s)| d s<\int_{x}^{\infty} s|q(s)| d s .
$$

If $k=i \nu$ is a zero of $y(\cdot, x)$ then $k^{2}=-\nu^{2}$ is a bound state of the Dirichlet Schrödinger operator $\mathbb{L}_{q}^{D}$ on $L^{2}(x, \infty)$. The only real zero of $y(\cdot, x)$ could be $k=0$. If $y(0, x)=0$ then $k^{2}=0$ is not a bound state of $\mathbb{L}_{q}^{D}$ on $L^{2}(x, \infty)$. The (full line) operator $\mathbb{L}_{q}$ has a bound state iff $y(0, x)=0$ for some $x$.

Note that the function $\int_{x}^{\infty}(s-x)|q(s)| d s$ is decreasing to zero and hence by Theorem $6.2 N_{x}=0$ for some $x$. This motivates

Definition 6.3. Let $q$ be subject to Hypothesis 2.1 (2). We call a number a large enough and denote $a>>1$ if $\int_{a}^{\infty}(x-a)|q(x)| d x<1$.

We will use Theorem 6.2 primarily in the form

Corollary 6.4. For some a large enough

$$
y(\cdot, x)^{ \pm 1} \in H^{\infty} \cap C .
$$

Since $q$ is real, $\overline{\psi_{ \pm}}$also solves (6.2) and one can easily see that the pairs $\left\{\psi_{+}, \overline{\psi_{+}}\right\}$ and $\left\{\psi_{-}, \overline{\psi_{-}}\right\}$form fundamental sets for (6.2). Hence $\psi_{\mp}$ is a linear combination of $\left\{\psi_{ \pm}, \overline{\psi_{ \pm}}\right\}$. Elementary Wronskian considerations then yield the (basic) right/left scattering relations

$$
T(k) \psi_{\mp}(x, k)=\overline{\psi_{ \pm}(x, k)}+R_{ \pm}(k) \psi_{ \pm}(x, k), k \in \mathbb{R},
$$

where $T, R_{ \pm}$, called the transmission and right/left reflection coefficients respectively. It immediately follows from (6.8) that $(\operatorname{Im} k=0)$

$$
\begin{aligned}
T & =\frac{2 i k}{W\left(\psi_{-}, \psi_{+}\right)}, \\
R_{+} & =\frac{W\left(\overline{\psi_{+}}, \psi_{-}\right)}{W\left(\psi_{-}, \psi_{+}\right)}, \quad R_{-}=\frac{W\left(\psi_{+}, \overline{\psi_{-}}\right)}{W\left(\psi_{-}, \psi_{+}\right)},
\end{aligned}
$$


where the Wronskians $\left(W(f, g)=f g^{\prime}-f^{\prime} g\right)$ are independent of $x\left(\partial_{x}\right.$ is missing in $\left.\mathbb{L}_{q}\right)$. By Theorem 6.1, $W\left(\psi_{-}, \psi_{+}\right)$is analytic in $\mathbb{C}^{+}$and by (6.1)

$$
W\left(\psi_{-}, \psi_{+}\right)=2 i k+o(1), k \rightarrow \infty, \operatorname{Im} k \geq 0 .
$$

Therefore $T(k)$ is analytic in $\mathbb{C}^{+}$except for zeros of $W\left(\psi_{-}, \psi_{+}\right)$and

$$
T(k)=1+o(1), k \rightarrow \infty, \operatorname{Im} k \geq 0 .
$$

If $k_{0}$ is a zero of $W\left(\psi_{-}, \psi_{+}\right)$then $\psi_{+}\left(x, k_{0}\right)=\mu_{0} \psi_{-}\left(x, k_{0}\right)$ (linearly dependent) with some $\mu_{0} \neq 0$, that occurs only for $k_{0} \in i \mathbb{R}_{+}$such that $k_{0}^{2}=-\kappa_{0}^{2}$, where $-\kappa_{0}^{2}$ is a bound state of $\mathbb{L}_{q}$. Next, from the well-known (and easily verifiable) formula $\partial_{k} W\left(\psi_{-}, \psi_{+}\right)=2 k \int \psi_{-} \psi_{+}$one has

$$
\left.\partial_{k} W\left(\psi_{-}, \psi_{+}\right)\right|_{k=i \kappa_{0}}=2 i \kappa_{0} \mu_{0}^{\mp 1} \int \psi_{ \pm}^{2}\left(\cdot, i \kappa_{0}\right),
$$

which means that $i \kappa_{0}$ is a simple zero of $W\left(\psi_{-}, \psi_{+}\right)$. It follows from (6.9) that

$$
\begin{aligned}
\operatorname{Res}_{i \kappa_{0}} T & =\left.\frac{2 i k}{\partial_{k} W\left(\psi_{-}, \psi_{+}\right)}\right|_{k=i \kappa_{0}}=i \mu_{0}^{ \pm 1}\left(\int \psi_{ \pm}^{2}\left(\cdot, i \kappa_{0}\right)\right)^{-1} \\
& =i \mu_{0}^{ \pm 1}\left\|\psi_{ \pm}\left(\cdot, i \kappa_{0}\right)\right\|_{2}^{-2} .
\end{aligned}
$$

The quantity

$$
c_{0}^{ \pm} \stackrel{\text { def }}{=}\left\|\psi_{ \pm}\left(\cdot, i \kappa_{0}\right)\right\|_{2}^{-2}
$$

is called the right/left norming constant of a bound state $-\kappa_{0}^{2}$. Its role in the IST is fundamental but we are not aware of its clear physical meaning.

The quantities $T, R_{ \pm},\left(\kappa_{n}, c_{n}^{ \pm}\right)$are called scattering and they can be obtained from the Jost solutions $\psi_{ \pm}$of $\mathbb{L}_{q} \psi=k^{2} \psi$. In other words, the scattering quantities can be read off the complete set of the spectral data of $\mathbb{L}_{q}$. We summarize the information about them in the following theorem.

Theorem 6.5. The transmission coefficient $T \in C$ and is analytic in $\mathbb{C}^{+}$except for a finite number of simple poles $\left\{i \kappa_{n}\right\}_{n=1}^{N}$ with the residues

$$
\operatorname{Res}\left(T, i \kappa_{n}\right)=i \mu_{n}^{ \pm 1} c_{n}^{ \pm},
$$

where $c_{n}^{ \pm}$are norming constants defined by (6.11) and $\mu_{n}$ determined from

$$
\psi_{+}\left(x, i \kappa_{n}\right)=\mu_{n} \psi_{-}\left(x, i \kappa_{n}\right) .
$$

Moreover,

$$
\lim T(k)=1, k \rightarrow \infty, \operatorname{Im} k \geq 0 .
$$

The reflection coefficients $R_{ \pm} \in C$ (but need not be analytic), $|R(k)|<1$ for $k \neq 0$ and generically $R(0)=-1$. Furthermore,

$$
T(-k)=\overline{T(k)}, \quad R_{ \pm}(-k)=\overline{R_{ \pm}(k)}, \quad|T(k)|^{2}+|R(k)|^{2}=1, \quad k \in \mathbb{R} .
$$

\footnotetext{
${ }^{10}$ I.e. $R(0)>-1$ only in exceptional cases and can be destroyed by a small perturbation.
} 
6.2. Inverse scattering problem. One asks what is the minimal subset of scattering quantities that determines $q$ completely?

The answer can be seen from the following arguments. Take one (e.g. right) of the basic scattering relations (6.8) and rewrite it in the form (recall (3.1)

$$
T y_{-}=\bar{y}_{+}+R \xi_{x} y_{+}
$$

Let us regard (6.15) as a Hilbert-Riemann problem of determining $y_{ \pm}$by given $T, R$ which we will solve by Hankel operator techniques. The potential $q$ can then be easily found by (6.3).

By Theorems 6.1, 6.5 Ty in (6.15) is meromorphic in $\mathbb{C}^{+}$with simple poles at $i \kappa_{n}$ and residences

$$
\begin{aligned}
\operatorname{Res}_{k=i \kappa_{n}} T(k) y_{-}(k, x) & =y_{-}\left(i \kappa_{n}, x\right) \operatorname{Res}_{k=i \kappa_{n}} T(k) \\
& =i \mu_{n} y_{-}\left(i \kappa_{n}, x\right) c_{n}^{+}=i c_{n}^{+} \xi_{x}\left(i \kappa_{n}\right) y\left(i \kappa_{n}, x\right),
\end{aligned}
$$

where we have used (6.12), (6.13). Note now that for each fixed $x$

$$
T(k) y_{-}(k, x)-1-\sum_{n=1}^{N} \frac{i c_{n} \xi_{x}\left(i \kappa_{n}\right)}{k-i \kappa_{n}} y\left(i \kappa_{n}, x\right) \in H^{2} .
$$

Abbreviating $R_{x}:=R \xi_{x}, c_{x, n}:=c_{n}^{+} \xi_{x}\left(i \kappa_{n}\right)$, rewrite (6.15) in the form

$$
\begin{aligned}
& T(k) y_{-}(k, x)-1-\sum_{n=1}^{N} \frac{i c_{x, n}}{k-i \kappa_{n}} y\left(i \kappa_{n}, x\right) \\
& =\overline{(y(k, x)-1)}+R_{x}(k)(y(k, x)-1) \\
& +R_{x}(k)-\sum_{n=1}^{N} \frac{i c_{x, n}}{k-i \kappa_{n}} y\left(i \kappa_{n}, x\right) .
\end{aligned}
$$

Noticing that the last term in (6.17) is in $H_{-}^{2}$, we can apply the Riesz projection $\mathbb{P}_{-}$to (6.17). Thus

$$
\mathbb{P}_{-}\left(\bar{Y}+R_{x} Y\right)+\mathbb{P}_{-} R_{x}-\sum_{n=1}^{N} i c_{x, n} \frac{Y\left(i \kappa_{n}, x\right)}{\cdot-i \kappa_{n}}-\sum_{n=1}^{N} \frac{i c_{x, n}}{\cdot-i \kappa_{n}}=0,
$$

where $Y:=y-1$. It is clear that $Y \in H^{2}$ for any $x \in \mathbb{R}$. Due to (6.4), $\bar{Y}=\mathbb{J} Y$ and by (4.3) we have

$$
\mathbb{P}_{-} \bar{Y}=\mathbb{P}_{-} \rrbracket Y=\mathbb{J} \mathbb{P}_{+} Y=\rrbracket Y .
$$

By (3.5)

$$
\sum_{n=1}^{N} i c_{x, n} \frac{Y\left(i \kappa_{n}, x\right)}{\cdot-i \kappa_{n}}=\mathbb{P}_{-} \sum_{n=1}^{N} i c_{x, n} \frac{Y(\cdot, x)}{\cdot-i \kappa_{n}} .
$$

Inserting (6.19) and (6.20) into (6.18), we obtain

$$
\mathbb{J} Y+\mathbb{P}_{-}\left(R_{x}-\sum_{n=1}^{N} \frac{i c_{x, n}}{\cdot-i \kappa_{n}}\right) Y=-\mathbb{P}_{-}\left(R_{x}-\sum_{n=1}^{N} \frac{i c_{x, n}}{\cdot-i \kappa_{n}}\right) .
$$

Applying $\mathbb{J}$ to both sides of this equation yields

$$
(\mathbb{I}+\mathbb{H}(\varphi)) Y=-\mathbb{H}(\varphi) 1,
$$


where $\mathbb{H}(\varphi)$ is the Hankel operator defined in Definition 4.1 with symbol

$$
\varphi(k)=\varphi_{x}(k)=R(k) \xi_{x}(k)+\sum_{n=1}^{N} \frac{c_{n} \xi_{x}\left(i \kappa_{n}\right)}{\kappa_{n}+i k}
$$

where $x$ is a real parameter $\left(\xi_{x}(k)=e^{2 i k x}\right)$.

Due to (6.14), $\mathbb{J} \varphi=\bar{\varphi}$ and hence by Proposition $4.5 \mathbb{H}(\varphi)$ is selfadjoint. Note that $\mathbb{H}(\varphi) 1$ on the right hand side of (6.21) should be interpreted as

$$
\mathbb{H}(\varphi) 1=\mathbb{P}_{+} \bar{\varphi} \in H^{2} .
$$

It is now clear that if we show that (6.21) is uniquely solvable and $Y(x, k)$ is its solution then the potential $q(x)$ can be found from (6.3) by

$$
q(x)=\partial_{x} \lim 2 i k Y(k, x), \quad k \rightarrow \infty .
$$

Thus, (6.21) suggests that what one needs to know to recover $q$ is the (right) reflection coefficient $R(k)$ for $k \geq 0$, bound states $\left\{-\kappa_{n}^{2}\right\}_{n=1}^{N}$ and their (right) norming constants $\left\{c_{n}\right\}_{n=1}^{N} 11$.

The set $S_{q}=\left\{R(k), k \geq 0,\left(\kappa_{n}, c_{n}\right)_{n=1}^{N}\right\}$ is called the (right) scattering data for $\mathbb{L}_{q}$. The solubility of (6.21) is equivalent to bounded invertibility of $\mathbb{I}+\mathbb{H}(\varphi)$.

6.3. Inverse scattering transform. To reformulate the classical IST in terms of Hankel operators, we recall the classical fact that the initial short range profile $q$ in (2.1)-(2.2) evolves under the KdV flow in such a way that the scattering data $S_{q}(t)$ for $q(x, t)$ evolves by (2.3). It is convenient to introduce

$$
S_{q}(x, t) \stackrel{\text { def }}{=}\left\{R(k) \xi_{x, t}(k), k \geq 0,\left(\kappa_{n}, c_{n} \xi_{x, t}\left(i \kappa_{n}\right)\right)_{n=1}^{N}\right\},
$$

the time evolved scattering data corresponding to the shifted initial profile $q(\cdot+x)$.

Observe that the KdV flow preserves at least the Schwartz class (an elementary well-known fact based on pure PDE techniques) and hence the inverse scattering procedure discussed in Subsection 6.2 also applies to the scattering data $S_{q}(x, t)$. It is remarkable that if one solves (6.21) with

$$
\varphi=\varphi_{x, t}(k)=R(k) \xi_{x, t}(k)+\sum_{n=1}^{N} \frac{c_{n} \xi_{x, t}\left(i \kappa_{n}\right)}{\kappa_{n}+i k}
$$

by the Fredholm series formula then $q(x, t)$ computed by (6.22) simplifies to

$$
q(x, t)=-2 \partial_{x}^{2} \log \operatorname{det}\left(\mathbb{I}+\mathbb{H}\left(\varphi_{x, t}\right)\right),
$$

where the determinant is understood in the classical Fredholm sense.

The formula (6.24) is a derivation of the well-known Dyson (also called Bargman or log-determinant) formula (see, e.g. [22, 67]).

We finally arrive at the following version of the classical IST

$$
q(x) \stackrel{(6.23)}{\longrightarrow} \mathbb{H}\left(\varphi_{x, t}\right) \stackrel{(6.24)}{\longrightarrow} q(x, t) .
$$

There has been nothing new in this section. Our derivation of (6.21) (its Fourier representation in the famous Gelfand-Levitan-Marchenko integral equation) consists of well-known classical components [61]. Even if (6.25) has not explicitly appeared in the literature before, it does not add much value to the classical IST. We shall use, however, (6.25) as a suitable starting point to extend IST far beyond

\footnotetext{
${ }^{11}$ Similarly, the left reflection coefficient $R_{-}$and left norming constants $\left\{c_{n}^{-}\right\}$in place of $R, c_{n}$.
} 
standard assumption (such as decay at infinity) on the initial data $q$. But much deeper understanding of the Hankel operator is required.

\section{The structure of the Reflection Coefficient}

To continue our program we shall understand the structure of reflection coefficient $R$ appearing in (6.23). We treat first the classical case and it will then be quite transparent how to generalize it.

7.1. The classical case. We consider the right reflection coefficient $R:=R_{+}$only. Recall our notation (3.1).

Proposition 7.1 (Structure of the classical reflection coefficient). Suppose $q$ is real and such that $(1+|x|) q(x) \in L^{1}$. Let $\left\{R,\left(\kappa_{n}, c_{n}\right)\right\}$ denote the scattering data. Then for some a large enough (in the sense of Definition 6.3) the (right) reflection coefficient $R$ can be split into

$$
R=A_{a}+r_{a} \xi_{a}^{-1} .
$$

The function $A_{a}$ is meromorphic in $\mathbb{C}^{+}$with the simple pole $\bigcup^{12}\left\{i \kappa_{n}\right\}_{n=1}^{N}$ and corresponding residues

$$
\operatorname{Res}\left(A_{a}, i \kappa_{n}\right)=i c_{n}
$$

and admits the representations

$$
\begin{aligned}
A_{a}(k) & =T(k) \frac{\psi_{-}(a, k)}{\psi_{+}(a, k)} \\
& =\xi_{a}^{-1}(k) S_{a}(k) / B(k),
\end{aligned}
$$

where $S_{a} \in H^{\infty} \cap C,\left\|S_{a}\right\|_{\infty} \leq 2$, and

$$
B(k)=\prod_{n=1}^{N} \frac{k-i \kappa_{n}}{k+i \kappa_{n}},
$$

is the (finite) Blaschke product with simple zeros at $\left\{i \kappa_{n}\right\}_{n=1}^{N}$. For $r_{a}$ we have

$$
r_{a}(k)=-\overline{y(k, a)} / y(k, a) \in C .
$$

Proof. From the right basic scattering relation (6.8) one has

$$
R(k)=T(k) \frac{\psi_{-}(k, a)}{\psi_{+}(k, a)}-\frac{\overline{\psi_{+}(k, a)}}{\overline{\psi_{+}(k, a)}}
$$

and by (6.5) equation (7.1) follows with $A_{a}$ and $r_{a}$ given by (7.3) and (7.4) respectively. By Theorems 6.1 and 6.5 $\psi_{ \pm}$and $T$ are analytic and hence $A_{a}$ is meromorphic in $\mathbb{C}^{+}$. Next, by Corollary 6.4 a number $a$ can be found so that $A_{a}(k)$ and $T(k) \psi_{-}(k, a)$ share the same poles. For the residues, by Theorem 6.5, one has

$$
\operatorname{Res}_{k=i \kappa_{n}} T(k) \frac{\psi_{-}(a, k)}{\psi_{+}(a, k)}=\frac{\psi_{-}\left(a, i \kappa_{n}\right)}{\psi_{+}\left(a, i \kappa_{n}\right)} \operatorname{Res}_{k=i \kappa_{n}} T(k)=\frac{1}{\mu_{n}} i \mu_{n} c_{n}=i c_{n} .
$$

Since $c_{n}>0, A_{a}(k)$ and $T(k)$ also share same poles.

Let us show (7.4). It is well-known [19] that

$$
T(k)=S(k) / B(k),
$$

\footnotetext{
${ }^{12} \operatorname{Recall}-\kappa_{n}^{2} \in \operatorname{Spec}\left(\mathbb{L}_{q}\right), n=1,2, \ldots, N$.
} 
where $B$ is given by (7.5) and

$$
S(k)=\exp \left\{\frac{1}{2 \pi i} \int \frac{\log \left(1-|R(s)|^{2}\right)}{s-k} d k\right\} .
$$

One can now easily see from Theorem 6.5 that $S \in H^{\infty} \cap C$ (even an outer function), and $\|S\|_{\infty} \leq 1$. The representation (7.4) follows from (7.3) with $S_{a}(k)=$ $S(k) y_{-}(k, a) / y_{+}(k, a)$. By Corollary 6.4, $S_{a} \in H^{\infty}$. It remains to estimate its $H^{\infty}$-norm. Due to (3.2) we can do it on the real line. By (7.4) and (7.1)

$$
\begin{aligned}
\left|S_{a}\right| & =\left|\xi_{a} B A_{a}\right|=\left|\xi_{a} B\left(R-r_{a} \xi_{a}^{-1}\right)\right| \\
& \leq\left|R-r_{a} \xi_{a}^{-1}\right| \leq 2 .
\end{aligned}
$$

Noticing that by (6.7) $r_{a} \in C$ concludes the proof.

Note that it is claimed in [59] (but no rigorous arguments are provided) that $R(k)$ can be analytically continued into the upper half plane under the only assumption that $\left(1+x^{2}\right) q \in L^{1}$. If it was true then $\psi_{+}(\cdot, a)$ would analytically continue into $\mathbb{C}_{-}$. The latter requires an exponential decay of $q$.

The remarkable feature of (7.1) is that while $A_{a}(k)$ does depend on $a$ but for $a>>1$ the poles of $A_{a}(k)$ occur only at the purely imaginary points $\left\{i \kappa_{n}\right\}_{n=1}^{N}$ such that $\left\{-\kappa_{n}^{2}\right\}_{n=1}^{N}$ is the set of bound states of $\mathbb{L}_{a}$, the residues of $A_{a}$ at $i \kappa_{n}$ being the norming constant $c_{n}$. This means that for $a>>1$ the function $A_{a}$ uniquely recovers the bound state information $\left(\kappa_{n}, c_{n}\right)$ and hence the knowledge of $\left\{R, A_{a}\right\}$ is equivalent to the knowledge of $\left\{R,\left(\kappa_{n}, c_{n}\right)\right\}$. Thus if we know (say) $\left.q\right|_{\mathbb{R}_{+}}$we can find $y(k, a)$ for $a>>1$ and hence $r_{a}(k)$ for any real $k$. One then computes $A_{a}$ by (7.1). We state what we have arrived at in two corollaries.

Corollary 7.2. The measure

$$
d \rho(s)=-i \sum_{n=1}^{N} \operatorname{Res}\left(A_{a}, i \kappa_{n}\right) \delta\left(s-\kappa_{n}\right) d s=\sum_{n=1}^{N} c_{n} \delta\left(s-\kappa_{n}\right) d s
$$

is independent of $a>>1$.

Corollary 7.3. For $a>>1$ the pair $\left\{R, A_{a}\right\}$ is a set of scattering data, i.e. it recovers the potential $q$ uniquely. Moreover, if $R$ and $\left.q\right|_{\mathbb{R}_{+}}$are known then $\left.q\right|_{\mathbb{R}_{-}}$is also known.

Remark 7.4. Proposition 7.1 is totally elementary but will nevertheless play a principal role in our considerations. It is worth mentioning that Corollary 7.3 (which we don't actually use) immediately implies many relevant results of [5, [6], [12], 37, 71] on the so-called inverse problems with partial information on the potential (see also [34] in this context).

7.2. The general step-like case. Through this subsection we deal with potentials subject to Hypothesis 2.1. We start with a brief review of Titchmarsh-Weyl theory of order two differential operators in dimension one (see, e.g. [76]).

A real-valued locally integrable potential $q$ is said to be Weyl limit point at $\pm \infty$ if the equation $\mathbb{L}_{q} u=\lambda u$ has a unique (up to a multiplicative constant) solution 13

\footnotetext{
${ }^{13}$ Note that Weyl solutions depend on the spectral parameter (energy) $\lambda$ while Jost solutions are typically considered as dependent on momentum $\sqrt{\lambda}$.
} 
$\Psi_{ \pm}(\cdot, \lambda) \in L^{2}(a, \pm \infty)$ for each $\lambda \in \mathbb{C}^{+}$. Such $\Psi_{ \pm}$is commonly called the Weyl solution on $(a, \pm \infty)$. The existence of $\Psi_{ \pm}$is directly related to the selfadjointness of $\mathbb{L}_{q}$ on $L^{2}(a, \pm \infty)$ with a Dirichlet (or any other selfadjoint) condition at $x=a \pm 0$. If $q \in L^{1}$ then the Weyl solutions $\Psi_{ \pm}(x, \lambda) \sim e^{ \pm i \sqrt{\lambda} x}, x \rightarrow \pm \infty$, clearly turn into Jost and we have

$$
\Psi_{ \pm}\left(x, k^{2}\right)=\psi_{ \pm}(x, k) .
$$

However Weyl solutions exist under much more general conditions on $q$ 's and no decay of any kind is required. There is no criterion for the limit point case in terms of $q$ (a major unsolved problem) but there are a number of sufficient conditions which are typically satisfied in most of realistic situations. For instance, any $q$ subject to Hypothesis 2.1 is in the limit point case at $\pm \infty$.

The following concept is fundamental in spectral theory of ordinary differential operators.

Definition 7.5 ( $m$-function). The function

$$
m_{ \pm}(\lambda, x)= \pm \frac{\partial_{x} \Psi_{ \pm}(x, \lambda)}{\Psi_{ \pm}(x, \lambda)}, \quad \lambda \in \mathbb{C}_{+}
$$

is called the (Dirichlet, principal) Titchmarsh-Weylm-function, or just m-function.

By definition $m_{ \pm}(\lambda, x)$ depends on two variables $(\lambda, x)$. The first one, energy, is the main variable. The other one is typically set $x=0$ with the convention $m_{ \pm}(\lambda, 0)=m_{ \pm}(\lambda)$. It is well-known that $m_{ \pm}(\cdot, x)$ is a Herglotz function. That is, it is analytic and maps $\mathbb{C}^{+}$to $\mathbb{C}^{+}$. The following general statement [3] is frequently used in spectral theory of ordinary differential operators.

Theorem 7.6 (Aronszajn-Donoghue, 1957). Let $f(\lambda)$ be a Herglotz function. Then

there exists a non-negative measure $d \mu$ such that

$$
f(\lambda)=a+b \lambda+\int \frac{1+\lambda s}{s-\lambda} \frac{d \mu(s)}{1+s^{2}}
$$

where

$$
a=\operatorname{Re} f(i), b \geq 0, \int \frac{d \mu(s)}{1+s^{2}}<\infty .
$$

Moreover, $\mu$ is computed by the Herglotz inversion formula

$$
\mu(\Delta)=\lim _{\varepsilon \rightarrow 0+} \frac{1}{\pi} \int_{\Delta} \operatorname{Im} f(s+i \varepsilon) d s .
$$

The formula (7.9) is called the Herglotz or Riesz-Herglotz representation. It is straightforward to derive from Theorem 7.6 the following

Corollary 7.7. If $f(\lambda)$ is Herglotz and $f(\lambda) \rightarrow 0$ as $\lambda \rightarrow \infty$ along any ray $0<$ $\varepsilon<\arg \lambda<\pi-\varepsilon$, and the support of $\mu$ in (7.9) is bounded from below then 7.9) reads

$$
f(\lambda)=\int \frac{d \mu(s)}{s-\lambda},
$$

where the measure $\mu$ is subject to $\int \frac{|s| d \mu(s)}{1+s^{2}}<\infty$. 
Titchmarsh-Weyl $m$-functions have many important properties. E.g. the classical Borg-Marchenko result says that the $m$-function determines the potential uniquely. This however is immaterial to us as apposed to the following convergence property which we state only for $m=m_{+}$.

Proposition 7.8. Let $q, q_{n}$ be in the limit point case at $+\infty$ and suppose that $q_{n} \rightarrow q$ in $L_{\mathrm{loc}}^{1}$. I.e. for any finite interval $I$

$$
\int_{I}\left|q-q_{n}\right| \rightarrow 0, n \rightarrow \infty
$$

Then

$$
m_{n} \rightrightarrows m, \quad n \rightarrow \infty, \text { in } \mathbb{C}^{+}
$$

and hence for the associated spectral measures of the half-line Dirichlet Schrödinger operators one has

$$
\mu_{n} \rightarrow \mu, \quad n \rightarrow \infty, \quad \text { weakly }
$$

We are not sure whom to attribute this statement. It appears in [13] as a part of a lemma 14. We learned it first from 64 but it may have been known much earlier as its proof rests on original ideas behind the limit point/limit circle classification.

The main convenience of $m$-function in our setting is that classical scattering theory can be extended far beyond strong decay assumptions at $\pm \infty$ if Jost solutions are suitably replaced with Weyl 33. For instance, one can formally define transmission and reflection coefficients merely by (6.9)-(6.10). Such generalizations, however, need not have suitable properties which could be a real problem.

Let us introduce now the right reflection coefficient for potentials subject to Hypothesis 2.1. Since $W\left(\psi_{+}, \overline{\psi_{+}}\right)=-2 i k$ the pair $\left\{\psi_{+}, \overline{\psi_{+}}\right\}$forms a fundamental set for $\mathbb{L}_{q} u=k^{2} u$ and hence the Weyl solution $\Psi_{-}$is a linear combination of $\left\{\psi_{+}, \overline{\psi_{+}}\right\}$. I.e. for any real $k \neq 0$

$$
T(k) \Psi_{-}\left(x, k^{2}\right)=\overline{\psi_{+}(x, k)}+R(k) \psi_{+}(x, k),
$$

holds with some $T$ and $R$. In analogy with (6.8) we call (7.12) the (right) basic scattering relation and similarly to (6.9) we introduce

Definition 7.9 (Reflection coefficient). We call

$$
R(k)=\frac{W\left(\overline{\psi_{+}}(\cdot, k), \Psi_{-}\left(\cdot, k^{2}\right)\right)}{W\left(\Psi_{-}\left(\cdot, k^{2}\right), \psi_{+}(\cdot, k)\right)}
$$

the (right) reflection coefficient.

Observe that since $W\left(\Psi_{-}, \psi_{+}\right)$is analytic in $\mathbb{C}^{+}$away from $k^{2} \in \operatorname{Spec}\left(\mathbb{L}_{q}\right) \cap \mathbb{R}_{-}$, the denominator $W\left(\Psi_{-}, \psi_{+}\right)$in (7.13) cannot vanish on a set of positive Lebesgue measure. Therefore $R$ is well defined by (7.13) for a.e. $k \in \mathbb{R}$. Similarly, $T$ is also well-defined by

$$
T(k)=\frac{2 i k}{W\left(\Psi_{-}\left(\cdot, k^{2}\right), \psi_{+}(\cdot, k)\right)} .
$$

Proposition 7.10 (Properties of the reflection coefficient). The reflection coefficient $R$ is symmetric $R(-k)=\overline{R(k)}$ and contractive $|R(k)| \leq 1$ a.e. Moreover, if $\sigma\left(\mathbb{L}_{q}\right)$ is the minimal support of the two fold a.c. spectrum of $\mathbb{L}_{q}$ then $|R(k)|<1$ for a.e. real $k$ such that $k^{2} \in \sigma\left(\mathbb{L}_{q}\right)$ and $|R(k)|=1$ otherwise.

\footnotetext{
${ }^{14}$ Stated there for $L_{\mathrm{loc}}^{2}$ as it was enough for the future purposes. The actual proof needs $L_{\text {loc }}^{1}$.
} 
Proof. From (7.12)-(7.12) and the Wronskian identity

$$
W_{12} W_{34}+W_{13} W_{42}+W_{14} W_{23}=0, W_{i k}:=W\left(f_{i}, f_{k}\right),
$$

omitting a straightforward computation, we have

$$
|R(k)|^{2}+\frac{|T(k)|^{2}}{2 i k} W\left(\Psi_{-}, \overline{\Psi_{-}}\right)\left(x, k^{2}+i 0\right)=1
$$

for any $x \in \mathbb{R}$ and a.e. $k \in \mathbb{R}$ or equivalently

$$
|R(k)|^{2}+\frac{\operatorname{Im} m_{-}\left(k^{2}+i 0, x\right)}{|k|} \cdot\left|T(k) \Psi_{-}\left(x, k^{2}+i 0\right)\right|^{2}=1 .
$$

It remains to notice that $\sigma\left(\mathbb{L}_{q}\right)$ coincides with the closure of $\left\{\operatorname{Im} m_{-}(t+i 0, x)>0\right\}$.

If $\Psi_{-}$is the Jost solution (e.g. the classical case) then $W\left(\psi_{-}(\cdot, k), \overline{\psi_{-}(\cdot, k)}\right)=$ $2 i k$ and (7.14) turns into $|R(k)|^{2}+|T(k)|^{2}=1$ as one would expect.

Consider the important case of $q(x) \rightarrow-h^{2}, x \rightarrow-\infty$, sufficiently fast. Then for any $k \in \mathbb{R}$

$$
\begin{gathered}
\Psi_{-}(x, k)=e^{-i \sqrt{k^{2}+h^{2}} x}+o(1), x \rightarrow-\infty, \\
\partial_{x} \Psi_{-}(x, k)+i \sqrt{k^{2}+h^{2}} \Psi_{-}(x, k)=o(1), x \rightarrow-\infty,
\end{gathered}
$$

and (17.14) implies

$$
|R(k)|^{2}+\sqrt{1+k^{2} / h^{2}}|T(k)|^{2}=1 .
$$

Remark 7.11. As opposed to the classical case, $\left\{\Psi_{-}, \overline{\Psi_{-}}\right\}$could be linearly dependent. In fact, $\left\{\Psi_{-}, \overline{\Psi_{-}}\right\}$are linearly dependent on the support of the set $\operatorname{Im} m_{-}\left(k^{2}+\right.$ $i 0, x)=0$. The latter may occur, e.g., if $q$ approaches $+\infty$ at $-\infty$ or for bounded $q$ without a specific pattern of behavior at $-\infty$ (e.g. the Gaussian white noise). The left basic scattering identity is then undefined for any $k$ but the right one remains defined. For a fairly complete description of different spectral regimes we refer to [33.

Here is the main statement of this section, which will be crucially used in the analysis of our Hankel operator.

Proposition 7.12 (Analytic split formula). For some $a>1$ (in the sense of Definition 6.3) the reflection coefficient can be represented as

$$
R=A_{a}(k)+r_{a}(k) \xi_{a}(k)^{-1}, \operatorname{Im} k=0,
$$

where

and $\left(\psi_{+}=: \psi\right)$

$$
r_{a}(k)=-\overline{y(k, a)} / y(k, a) \in C,
$$

$$
A_{a}(k)=\frac{1}{\psi(a, k)^{2}} \frac{2 i k}{m_{+}\left(k^{2}, a\right)+m_{-}\left(k^{2}, a\right)} .
$$

The function $A_{a}$ is analytic in $\mathbb{C}^{+}$except for

$$
i \Delta=\left\{k \in i \mathbb{R}_{+}: k^{2} \in \operatorname{Spec}\left(\mathbb{L}_{q}\right) \cap \mathbb{R}_{-}\right\}
$$

and

$$
\left|A_{a}(k)\right| \leq 2 \text { for a.e. } k \in \mathbb{R}
$$


Furthermore, for the jump $A_{a}(i s-0)-A_{a}(i s+0)$ across $i \Delta$ we have

$$
\begin{aligned}
& i\left(A_{a}(i s-0)-A_{a}(i s+0)\right) d s / 2 \pi=\psi(a, i s)^{-2} d \mu_{a}\left(-s^{2}\right) \\
& =: \quad d \rho(s),
\end{aligned}
$$

where

$$
d \mu_{a}(\lambda)=-\frac{1}{\pi} \operatorname{Im}\left[m_{+}(\lambda+i 0, a)+m_{-}(\lambda+i 0, a)\right]^{-1} d \lambda .
$$

The measure $d \rho$ is non-negative, finite, supported on $\Delta$, and independent of a.

Proof. The split (7.16) is obtained same way as (7.1). By (7.13) and (7.8) we have

$$
R(k)=\frac{1}{\psi(a, k)^{2}} \frac{2 i k}{m_{+}\left(k^{2}, a\right)+m_{-}\left(k^{2}, a\right)}-\frac{\overline{\psi(a, k)}}{\psi(a, k)} \xi_{a}^{-1}(k)
$$

which proves (7.16) with $A_{a}$ given by (7.18). The bound (7.19) follows from Proposition 7.10 and the obvious fact $\left|r_{a} \xi_{a}{ }^{-1}\right|=1$.

Note that since $m_{ \pm}$are both Herglotz, the function

$$
f_{a}(\lambda)=-\left(m_{+}(\lambda, a)+m_{-}(\lambda, a)\right)^{-1}
$$

is also Herglotz. It follows from (7.10) that its representing measure $d \mu_{d} \sqrt{15}$, given by (7.21), is non-negative, finite $\left(\int \frac{d \mu}{1+t^{2}}<\infty\right)$ and supported [76] on the spectrum of $\mathbb{L}_{q}$. Now, from (7.18) and (7.21)

$$
\begin{aligned}
& i\left(A_{a}(i s+0)-A_{a}(i s-0)\right) d s / 2 \pi \\
& =-\frac{1}{\pi} \operatorname{Im} A_{a}(i s+0) d s=\frac{1}{\psi^{2}(a, i s)} \frac{1}{\pi} \operatorname{Im} \frac{-(-2 s) d s}{m_{+}\left(-s^{2}+i 0, a\right)+m_{-}\left(-s^{2}+i 0, a\right)} \\
& =\psi(a, i s)^{-2} d \mu_{a}\left(-s^{2}\right)=: d \rho_{a}(s)
\end{aligned}
$$

and (7.20) follows. By Corollary 6.4 $\psi(a, i s)^{-2}$ is bound for $a>>1$ and one concludes that the measure $d \rho(s)$ is finite. It remains to show that $\rho$ is independent of $a$. To show this we employ the following approximation arguments. Consider $q_{b}:=\chi_{b} q$ with $b<a$. (e.g. $q_{b}(x)=0, x<b$ and $q_{b}(x)=q(x), x \geq b$ ). Then by Proposition $7.8 m_{-}^{b}(\lambda, a) \rightrightarrows m_{-}(\lambda, a)$ in $\mathbb{C}^{+}$as $b \rightarrow-\infty$ and hence $A_{a b} \rightrightarrows A_{a}$ in $\mathbb{C}^{+} \backslash i \Delta$. Therefore, $A_{a b} \rightarrow A_{a}$ weakly on the boundary of $\mathbb{C}^{+} \backslash i \Delta$. Apparently

$$
d \rho_{a b}(s)=\sum_{n=1}^{N_{b}} c_{n}^{b} \delta\left(s-\kappa_{n}^{b}\right) d s,
$$

where $\left\{-\left(\kappa_{n}^{b}\right)^{2}\right\}$ are the bound states of $\mathbb{L}_{q_{b}}$ and $c_{n}^{b}$ are their norming constants, is, by Corollary 7.2 independent of $a$. That is, $\rho_{a b}=\rho_{b}$. But $\rho(s)=w-\lim \rho_{b}(s)$ as $b \rightarrow-\infty$ which concludes the proof.

Note that the measure $\rho$ plays the role of 'smeared bound states norming constants' and can be recovered from $A_{a}$ by (7.20). This is the main value of our split (7.16). This split has a few alternative forms. E.g. $R=\left(A_{a}+\xi_{a}^{-1}\right)+\left(r_{a}-1\right) \xi_{a}^{-1}$ also splits $R$ into an analytic function and a small remainder. Such split (given

\footnotetext{
${ }^{15}$ Through the paper we use the convention

$$
\operatorname{Im} f(t+i 0) d t:=w-\lim _{\varepsilon \rightarrow+0} \operatorname{Im} f(t+i \varepsilon) d t .
$$
}


in a different form) was crucially used in our 68. The proof given here appears particularly short.

We find the next consequence of Proposition 7.12 quite surprising.

Corollary 7.13. If the negative spectrum of $\mathbb{L}_{q}$ is discrete then the sequence $\left\{c_{n}\right\}$ of the right norming constants is summable.

The next statement offers some more information on the components in (7.16).

Proposition 7.14 (More properties of the analytic split). The function $r_{a} \xi_{a}{ }^{-1}$ in (7.16) can further be split into

$$
r_{a}(k)=\xi_{a}(k) R_{a}(k)-y(k, a)^{-1} T_{a}(k),
$$

where $T_{a}, R_{a}$ are the transmission and reflection coefficients for $q_{a}=\chi_{a} q$. We have

$$
R_{a}(k)=O(1 / k), k \rightarrow \pm \infty ; R_{a}(k)=o(1 / a), a \rightarrow \infty,
$$

and $T_{a}$ has at most one pole $\kappa_{a}$ subject to

$$
\kappa_{a}=o(1 / a), \quad a \rightarrow \infty .
$$

The function $A_{a}$ in (7.16) has the property: for $C$ large enough

$$
\left|\xi_{a}(k) A_{a}(k)\right| \lesssim_{a, q} 1 \text { for }|k| \geq C, \operatorname{Im} k \geq 0 .
$$

If $\Delta=\left\{\kappa_{n}\right\} \in l^{1}$ then $A_{a}$ has the form similar to 7.4

$$
A_{a}(k)=\xi_{a}^{-1}(k) S_{a}(k) / B(k)
$$

where $S_{a} \in H^{\infty},\left\|S_{a}\right\|_{\infty} \leq 2$, and

$$
B(k)=\prod_{n \geq 1} \frac{k-i \kappa_{n}}{k+i \kappa_{n}} .
$$

Proof. Equation (7.22) is nothing but rearranged (7.7) written for $q_{a}$. The asymptotics (7.23) directly follow from Theorem 6.1. The asymptotics (7.24) holds due to the Thirring-Lieb inequality

$$
\kappa_{a} \lesssim \int_{a}^{\infty}|q|<\frac{1}{a} \int_{a}^{\infty} x|q(x)| d x .
$$

The rest of the statement is a bit harder. It follows from (7.18) that

$$
\xi_{a} A_{a}=y_{a}^{-2} g_{a},
$$

where

$$
y_{a}(k):=y(k, a), \quad g_{a}(k):=\frac{2 i k}{m_{+}\left(k^{2}, a\right)+m_{-}\left(k^{2}, a\right)} .
$$

By Corollary 6.4, (7.25) is then equivalent to $\left|g_{a}(k)\right| \lesssim_{a, q} 1$ for $|k| \geq C, \operatorname{Im} k \geq 0$. The Atkinson classical result [4] says that $m_{ \pm}\left(k^{2}, a\right)=i k+o(1)$ as $|k| \rightarrow \infty, 0<$ $\varepsilon<\arg k<\pi-\varepsilon$, which by Corollary 7.7 implies that

$$
\begin{aligned}
g_{a}(\lambda) & =2 i k \int \frac{d \mu_{a}(s)}{k^{2}-s}=g_{a}^{+}(k)+g_{a}^{-}(k), \\
g_{a}^{ \pm}(k) & :=2 i k \int_{\mathbb{R}_{ \pm}} \frac{d \mu_{a}(s)}{k^{2}-s}
\end{aligned}
$$

where $d \mu_{a}$ is given by (7.21), and

$$
g_{a}(\lambda)=1+o\left(k^{-1}\right),|k| \rightarrow \infty, 0<\varepsilon<\arg k<\pi-\varepsilon .
$$


By Proposition 7.12, $\operatorname{Supp}\left(\mu_{a}\right) \cap \mathbb{R}_{-} \subseteq\left[-h_{0}^{2}, 0\right]$ and we clearly have

$$
g_{a}^{-}(k)=2 i k \int_{-h_{0}^{2}}^{0} \frac{d \mu_{a}(s)}{k^{2}-s}=O\left(\frac{1}{k}\right),|k| \rightarrow \infty .
$$

Thus we are done if we show

$$
G_{a}(k):=\frac{k}{k+i} g_{a}^{+}(k) \in H^{\infty} .
$$

To this end consider, as before, $q_{b}=q \chi_{b}$ first. We are now under conditions of the previous subsection and Proposition 7.1]applies. In particular, (in obvious notation)

$$
g_{a b}(k)=\psi(a, k)^{2} A_{a b}(k)=y_{a}(k)^{2} S_{a b}(k) / B_{b}(k),
$$

where $B_{b}$ is the (necessarily finite) Blaschke product with zeros at $\left\{i \kappa_{n}^{b}\right\}, y_{a}^{2} S_{a b} \in$ $H^{\infty} \cap C$, and uniformly in $b<a$

$$
\left\|y_{a}^{2} S_{a b}\right\|_{\infty} \leq 2\left\|y_{a}\right\|_{\infty}^{2} \lesssim_{q_{a}} 1
$$

One concludes from the representation

$$
G_{a b}(k)=\frac{k}{k+i} g_{a b}(k)-\frac{k}{k+i} g_{a b}^{-}(k)
$$

that $G_{a b}$ is analytic in $\mathbb{C}^{+}$. Moreover, since $g_{a b}^{-}$is a rational function with poles at $\left\{ \pm i \kappa_{n}^{b}\right\}, G_{a b}$ is continuous on $\mathbb{R}$ and it follows from (7.32), (7.31), (7.29), and Proposition 7.1 that

$$
\begin{aligned}
\lim G_{a b}(k) & =\lim g_{a b}(k)-\lim g_{a b}^{-}(k) \\
& =1,|k| \rightarrow \infty, \operatorname{Im} k \geq 0 .
\end{aligned}
$$

Thus, by the Phragmén-Lindelöf principle $G_{a b} \in H^{\infty} \cap C$ for any $b<a$ and on the real line, uniformly in $b$ (for $|b|$ large), we have

$$
\left\|G_{a b}\right\|_{\infty} \leq 4\left\|y_{a}\right\|_{\infty}^{2}+\sup _{k \in \mathbb{R}}\left|\frac{2 k^{2}}{k+i} \int_{\mathbb{R}_{-}} \frac{d \mu_{a b}(s)}{k^{2}-s}\right| \lesssim a, q 1 .
$$

Here we have used the fact that due to Proposition 7.8, $\mu_{a}(s)=w-\lim _{b \rightarrow-\infty} \mu_{a b}(s)$. By the same proposition, uniformly in $\mathbb{C}^{+}$

$$
\begin{aligned}
G_{a b}(k) & =\frac{k}{k+i}\left(\psi(a, k)^{2} A_{a b}(k)-2 i k \int_{\mathbb{R}_{-}} \frac{d \mu_{a b}(s)}{k^{2}-s}\right) \\
& \rightrightarrows \underset{b \rightarrow-\infty}{\rightrightarrows} \frac{k}{k+i}\left(\psi(a, k)^{2} A_{a}(k)-2 i k \int_{\mathbb{R}_{-}} \frac{d \mu_{a}(s)}{k^{2}-s}\right) \\
& =G_{a}(k)
\end{aligned}
$$

which combined with (7.33) proves (7.30).

It remains to show (7.26). A bit more complicated approximation of $q$ is required. Split

$$
q=q_{+}-q_{-} \text {where } q_{ \pm}:= \pm \frac{1}{2}(q \pm|q|) \geq 0 .
$$

and consider $q_{b c}=\chi_{c} q_{+}-\chi_{b} q_{-}$with $b, c<a$. Clearly

$$
q_{b c} \underset{c \rightarrow-\infty}{\longrightarrow} q_{b} \underset{b \rightarrow-\infty}{\longrightarrow} q \text { in } L_{\mathrm{loc}}^{1} \text {. }
$$


Then by Proposition $7.8 m_{-}^{b c}(\cdot, a) \underset{c \rightarrow-\infty}{\rightrightarrows} m_{-}^{b}(\cdot, a) \underset{b \rightarrow-\infty}{\rightrightarrows} m_{-}(\cdot, a)$ in $\mathbb{C}^{+}$and hence

$$
A_{a b c} \underset{c \rightarrow-\infty}{\rightrightarrows} A_{a b} \underset{b \rightarrow-\infty}{\rightrightarrows} A_{a} \text { in } \mathbb{C}^{+} .
$$

Since $q_{b c}$ is clearly subject to Proposition 7.1, the representation (7.4) is valid. We are done then if we show that

$$
B_{b c} \underset{c \rightarrow-\infty}{\rightrightarrows} B_{b} \underset{b \rightarrow-\infty}{\rightrightarrows} B \text { in } \mathbb{C}^{+}
$$

as (7.34) and (7.35) will immediately imply that the limit $\lim _{b \rightarrow-\infty} \lim _{c \rightarrow-\infty} S_{a b c}$ exists on compacts in $\mathbb{C}^{+}$and defines an $H^{\infty}$ function $S_{a}$ satisfying $\left\|S_{a}\right\|_{\infty} \leq 2$.

We make use of a well-known general perturbation principle which in our particular case, loosely speaking, says that the (negative) bound states of $\mathbb{L}_{q_{b c}}$ move in unison rightward (leftward) as $c(b)$ moves leftward and new bound states may disappear at (appear from) 0 only. Together with Proposition 7.8 this means that the (finite) Blaschke product $B_{b c}$ converges to a finite Blaschke product $B_{t}{ }^{16}$ and, in turn, by Proposition $3.1 B_{b}$ converges to the (infinite) Blaschke product $B$ given by (7.27).

We conclude this section with the following explicitly solvable case which appears illustrative.

Example 7.15. If $q(x)$ is a pure step function, i.e. $q(x)=-h^{2}, x<0, q(x)=$ $0, x \geq 0$ then $\operatorname{Spec}\left(\mathbb{L}_{q}\right)=\left(-h^{2}, \infty\right)$ and purely a.c., $\left(-h^{2}, 0\right)$ and $(0, \infty)$ being its simple and two fold components respectively. Moreover

$$
R(k)=-\left(\frac{h}{\sqrt{k^{2}}+\sqrt{k^{2}+h^{2}}}\right)^{2}, \quad d \rho(s)=\frac{2 s}{\pi h^{2}} \sqrt{h^{2}-s^{2}} d s .
$$

The function $y(\cdot, x)^{-1} \in H^{\infty}$ for any $x>-\frac{\pi}{2 h}$.

\section{The IST Hankel Operator}

The previous section suggests that the Hankel operator arising in the IST has a very specific structure. In this section we state and prove some of its properties of principal importance.

Definition 8.1 (IST Hankel operator). Assume that initial data $q$ is subject to Hypothesis [2.1. Let $R$ and $\rho$ be as in Definition 7.9 and Proposition 7.12 respectively. We call the Hankel operator

$$
\mathbb{H}(x, t):=\mathbb{H}\left(\varphi_{x, t}\right)
$$

with the symbol

$$
\varphi_{x, t}(k)=\xi_{x, t}(k) R(k)+\int_{0}^{h_{0}} \frac{\xi_{x, t}(i s) d \rho(s)}{s+i k},
$$

the IST Hankel operator associated with $q$.

Here is the main result of this section

\footnotetext{
${ }^{16} B_{b}(k)$ could be 1.
} 
Theorem 8.2 (Fundamental properties of the IST Hankel operator). Under Hypothesis 2.1 the IST Hankel operator $\mathbb{H}(x, t)$ is well-defined and has the properties: for any $x \in \mathbb{R}, t>0$

(1) $\mathbb{H}(x, t)$ is selfadjoint,

(2) $\mathbb{H}(x, t)$ is compact,

(3) $\mathbb{I}+\mathbb{H}(x, t)>0$.

Proof. Without loss of generality we assume $a=0$. Consider the principal part (see (4.8)) of $\xi_{x, t} A_{0}$ :

$$
\left(\widetilde{\mathbb{P}}_{-} \xi_{x, t} A_{0}\right)(k)=-\frac{1}{2 \pi i} \int\left(\frac{1}{\lambda-(k-i 0)}-\frac{1}{\lambda+i)}\right) \xi_{x, t}(\lambda) A_{0}(\lambda) d \lambda
$$

Deform the contour of integration to $R+i h, h>h_{0}$. It can be easily justified due to (7.25) and the rapid decay of $\xi_{x, t}(\lambda)$ if $|\lambda| \rightarrow \infty$ along $R+i h$ for arbitrary $h>0$.

So, we have

$$
\begin{aligned}
\left(\widetilde{\mathbb{P}}_{-} \xi_{x, t} A_{0}\right)(k)= & -\frac{1}{2 \pi i} \int_{\mathbb{R}+i h} \frac{\xi_{x, t}(\lambda) A_{0}(\lambda)}{\lambda-k} d \lambda-\int_{\mathbb{R}+i h} \frac{\xi_{x, t}(\lambda) A_{0}(\lambda)}{\lambda+i} d \lambda \\
& -\int_{0}^{h_{0}} \frac{\xi_{x, t}(i s)}{s+i k} d \rho(s)-\frac{1}{i} \int_{0}^{h_{0}} \frac{\xi_{x, t}(i s) d \rho(s)}{s+1} .
\end{aligned}
$$

It is easy to see that the function

$$
\Phi(k):=-\frac{1}{2 \pi i} \int_{\mathbb{R}+i h} \frac{\xi_{x, t}(\lambda) A_{0}(\lambda)}{\xi-k} d \lambda
$$

belong to $C$. Since $\xi_{x, t} A_{0} \in L^{\infty}$ the third term in (8.2)

$$
\phi_{x, t}(k):=\int_{0}^{h_{0}} \frac{\xi_{x, t}(i s)}{s+i k} d s
$$

belongs to BMO and by Proposition 4.4 operator $\mathbb{H}(x, t)$ is well-defined and bounded. Statement (1) follows then from Propositions 4.5 and 7.10.

To prove (2) we observe (see (8.1), Theorem 4.3 and (4.10) ) that

$$
\mathbb{H}(x, t)=\mathbb{H}\left(\Phi+r_{0} \xi_{0}^{-1}\right) .
$$

Since $\Phi \in C$ and $r_{0} \in C$ (due to (7.17) $)$ we see that Theorem 5.2 implies statement (2).

We are ready now to prove part (3). Let $f \in H^{2}$, then

$$
\langle(I+\mathbb{H}(x, t)) f, f\rangle=\langle f, f\rangle+\left\langle\mathbb{H}\left(\xi_{x, t} R\right) f, f\right\rangle+\left\langle\mathbb{H}\left(\phi_{x, t}\right) f, f\right\rangle,
$$

where $\phi_{x, t}$ is given by (8.3). Since $\phi_{x, t} \in \mathrm{BMO}$ the last term of (8.4) exists and

$$
\begin{aligned}
\left\langle\mathbb{H}\left(\phi_{x, t}\right) f, f\right\rangle & =-i \int_{0}^{h_{0}} d \rho(s)\left\langle J \mathbb{P}_{-} \frac{f}{\cdot-i s}, f\right\rangle \\
& =i \int_{0}^{h_{0}} d \rho(s) f(i s)\left\langle\frac{1}{\cdot+i s}, f\right\rangle=2 \pi \int_{0}^{h_{0}}|f(i s)|^{2} d \rho(s) \geq 0 .
\end{aligned}
$$

Since $\left\|\xi_{x, t} R\right\|_{\infty} \leq 1$ we have

$$
\left\langle\left(I+\mathbb{H}\left(\xi_{x, t} R\right)\right) f, f\right\rangle \geq 0 .
$$

Suppose that (3) does not hold. Then (8.5) and (8.6) imply

$$
\int_{0}^{h_{0}}|f(i s)|^{2} d \rho(s)=0 .
$$


We need to consider three cases.

Case 1. The support of $\rho$ is a uniqueness set for $H^{2}$. Then (8.7) implies that $f \equiv 0$ and statement (3) trivially follows.

Let now Supp $\rho$ be a non uniqueness set for $H^{2}$. Then $\operatorname{Supp} \rho=\left\{i \kappa_{n}\right\}$ with $\kappa_{n}>0$ subject to the Blaschke condition and condition (8.7) holds iff $f\left(i \kappa_{n}\right)=0$. It follows from the canonical factorization theorem that $f=B F$, where $B$ is the Blaschke product with zeros $\left\{i \kappa_{n}\right\}$ and $F \in H^{2}$. Thus (8.4) reads

$$
\langle(I+\mathbb{H}(x, t)) f, f\rangle=\langle F, F\rangle+\left\langle\mathbb{H}\left(\xi_{x, t} B R\right) F, B F\right\rangle .
$$

By Proposition 7.14 (see 7.26) $) B A_{0} \in H^{\infty}$. Moreover, by Proposition 7.12 $r_{0} \xi_{0}^{-1} \in C$ and by Theorem $5.3 \xi_{x, t} \in H^{\infty}+C$. Thus we have $\xi_{x, t} B R \in H^{\infty}+C$.

Case 2. Supp $\rho$ is not a uniqueness set and $R$ is not unimodular function. By Theorem 5.10 we have

and (8.9) implies

$$
\left\|\mathbb{H}\left(\xi_{x, t} B R\right)\right\|<1
$$

$$
\left|\left\langle\mathbb{H}\left(\xi_{x, t} B R\right) F, B F\right\rangle\right| \leq\left\|\mathbb{H}\left(\xi_{x, t} B R\right)\right\| \cdot\|F\|_{2}\|B F\|_{2}<\|F\|_{2}^{2},
$$

which immediately yields statement (3).

Case 3. Supp $\rho$ is not a uniqueness set and $R$ is a unimodular function. Then by Theorem 5.3 (see (5.3) $) \xi_{x, t}=B_{x, t} u_{x, t}$ with some infinite Blaschke product $B_{x, t}$ and unimodular function $u_{x, t}$ from $C$. Therefore $\xi_{x, t} B R \in H^{\infty}+C$ and by Lemma $5.6 \xi_{x, t} B R$ is not invertible in $H^{\infty}+C$. By Theorem 5.8 then (8.9) holds and as in Case 2 statement (3) follows.

Remark 8.3. Theorem 8.2 says that $(\mathbb{I}+\mathbb{H}(x, t))^{-1}$ is a bounded operator for any $x \in \mathbb{R}$ and $t>0$, which is of course of a particular importance for validation of the IST. Cases 1,2 in the proof are easy and were done in [69. Case 3 is much more subtle. Under assumption that $h_{0}=0$ in Hypothesis 2.1 and $q_{a}=0$ it was proven in our [40. Then in [41] we relaxed the condition $h_{0}=0$ but imposed some extra conditions on the negative spectrum of $\mathbb{L}_{q}$. In the full generality Theorem 8.2 appears first in this paper and is one of our main results.

Remark 8.4. Theorem 8.2 does not say that if we split $\mathbb{H}(x, t)$ into two Hankel operators corresponding to the two pieces in (8.1) then each Hankel operator is compac17. However, if we notice that $\mathbb{H}\left(\phi_{x, t}\right)$ is unitary equivalent to the integral operator 4.12) on $L^{2}\left(\mathbb{R}_{+}\right)$with the continuous kernel $h(\cdot)=\int_{0}^{h_{0}} e^{-s(\cdot)} \xi_{x, t}(i s) d \rho(s)$ then

$$
\operatorname{tr} \mathbb{H}\left(\phi_{x, t}\right)=\int_{0}^{\infty} d z \int_{0}^{h_{0}} e^{-2 z s} \xi_{x, t}(i s) d \rho(s)=\frac{1}{2} \int_{0}^{h_{0}} \xi_{x, t}(i s) \frac{d \rho(s)}{s},
$$

which means that $\mathbb{H}\left(\phi_{x, t}\right) \in \mathfrak{S}_{1}$ iff $\int_{0}^{h_{0}} d \rho(s) / s$ is bounded. It is clearly the case in Example 7.15 but untrue in general. Exploiting the same unitary equivalence argument, one can easily prove that $\mathbb{H}\left(\phi_{x, t}\right)$ is bounded iff $\rho$ is a Carleson measure, i.e.

$$
\sup \left\{\frac{1}{\delta} \int_{0}^{\delta} d \rho: \delta>0\right\}<\infty .
$$

We find this result quite interesting as conditions like (8.10) are frequently a priori assumed even in the case when $q$ tends to a constant at $-\infty$ (c.f. [78]). Also note

\footnotetext{
${ }^{17}$ Some subtle conditions for $\mathbb{H}\left(\phi_{x, t}\right) \in \mathfrak{S}_{\infty}$ are studied in our 41 .
} 
that 8.10) means that 0 must not be an eigenvalue of $\mathbb{L}_{q}$. Of course, it follows from (7.30) that there are no positive (imbedded) bound states either. Thus, Hypothesis 2.1 imposes a restriction on the spectrum: the discrete spectrum of $\mathbb{L}_{q}$ could only be negative.

\section{Singular numbers of the IST Hankel operator}

As well-known a bounded operator $\mathbb{A}$ is compact $\left(\mathbb{A} \in \mathfrak{S}_{\infty}\right)$ if it can be uniformly approximated by rank $n$ operators $\mathbb{A}_{n}$. Singular numbers $s_{n}(\mathbb{A})$ give an accurate quantitative description of the rate of convergence $\mathbb{A}_{n} \rightarrow \mathbb{A}$. In the context of Hankel operators singular numbers gain a whole new meaning as $s_{n}(\mathbb{H}(\varphi))$ are directly related to best rational approximations of $\varphi$. Consequently, since about 1970, sparked by seminal works due to Adamyan-Arov-Krein, a large variety of issues related to singular number 18 have been extensively studied (see, e.g. [46, 66. and the extensive literature cited therein). We however are not sure if any of these has been used in soliton theory. In this section we shall demonstrate how the Adamyan-Arov-Krein classical theory beautifully yields subtle relations between the decay of $s_{n}(\mathbb{H}(x, t))$ and properties of the initial data $q$. In the subsequent section this will be translated into substantial conclusions on the initial value problem for the KdV equation. Due to space limitations, we focus on the opportunities that the theory of Hankel operators promises rather than completeness of our results.

9.1. Some general statements on singular numbers of Hankel operators. Let $\mathcal{R}_{n}$ denote the set of rational functions bounded at infinity with all poles in the upper half plane of total multiplicity $\leq n$. The following theorems are fundamental in the study of singular numbers of Hankel operators.

Theorem 9.1 (Adamyan-Arov-Krein, 1971). Let $\varphi \in L^{\infty}$. Then

$$
s_{n}(\mathbb{H}(\varphi))=\operatorname{dist}_{L^{\infty}}\left(\varphi, \mathcal{R}_{n}+H^{\infty}\right) .
$$

Theorem 9.2 (Bernstein-Jackson, 1910). Let $\varphi \in C^{m}$. Then

$$
\operatorname{dist}_{L^{\infty}}\left(\varphi, \mathcal{R}_{n}+H^{\infty}\right) \lesssim\left\|\varphi^{(m)}\right\|_{\infty} / n^{m}
$$

We will need a simple

Lemma 9.3. Let $C, b>0, p \geq 1$ and $\left\{s_{n}\right\}_{n \geq 1}$ be a positive sequence. If

$$
s_{n} \leq \frac{C(m !)^{p}}{b^{m}} \frac{1}{n^{m}}, \quad \forall m \in \mathbb{N}_{0}
$$

then

$$
s_{n} \leq C 2^{p} \exp \left\{-(p / 2)(b n)^{1 / p}\right\} .
$$

Proof. Without loss of generality we may set $C=1=b$. Multiplying $\frac{n^{m}}{(m !)^{p}} s_{n} \leq 1$ by $2^{-m}$ and then summing on $m \geq 0$ yields

$$
s_{n} \sum_{m \geq 0} \frac{n^{m}}{(m !)^{p}} \frac{1}{2^{m}} \leq \sum_{m \geq 0} \frac{1}{2^{m}}=2 .
$$

\footnotetext{
${ }^{18}$ In particular, membership in $\mathfrak{S}_{p}$ classes.
} 
Bound now the left hand side of (9.3) from below by the Jensen inequality:

$$
\begin{aligned}
s_{n} \sum_{m \geq 0} \frac{n^{m}}{(m !)^{p}} \frac{1}{2^{m}} & \geq s_{n} 2^{1-p}\left(\sum_{m \geq 0} \frac{\left(n^{1 / p} / 2\right)^{m}}{m !}\right)^{p} \\
& =s_{n} 2^{1-p}\left(\exp \left(n^{1 / p} / 2\right)\right)^{p} .
\end{aligned}
$$

Combining (9.2) and (9.4) proves the lemma.

Theorems $9.1,9.2$ and Lemma 9.3 immediately yield the following observation.

Proposition 9.4. Let $f \in L^{1}, h>0$ and

$$
\varphi(k)=\int \frac{f(s)}{s+i h-k} d s
$$

Then

$$
s_{n}(\mathbb{H}(\varphi)) \leq(2 / h)\|f\|_{1} \exp \{-(h / 2) n\} .
$$

We conclude this subsection with a useful

Remark 9.5. Due to the well-known Ky Fan inequality

$$
s_{n+m-1}(\mathbb{A}+\mathbb{B}) \leq s_{n}(\mathbb{A})+s_{m}(\mathbb{B}),
$$

a finite rank perturbation has no effect on asymptotics of singular numbers. By Theorem $9.1 \mathbb{H}(\varphi)$ is finite rank iff $\varphi$ is rational. This means that the rational part of a symbol $\varphi$ does not influence the asymptotics of $s_{n}(\mathbb{H}(\varphi)) \quad n \rightarrow \infty$.

9.2. Rate of decay of singular numbers of the IST Hankel operator . With the preparatory material out of the way, we turn now to the actual results of this section. The following easily verifiable formula will be used $(t>0)$

$$
\left|\xi_{x+i y, t}(\alpha+i \beta)\right|=\xi_{x, t}(i \beta) \exp \left\{-\left(\sqrt{24 \beta t} \alpha+\frac{y}{\sqrt{24 \beta t}}\right)^{2}+\frac{y^{2}}{24 \beta t}\right\} .
$$

Theorem 9.6 (Asymptotics of singular numbers). Assume Hypothesis [2.1] with $w(x)=\exp \left(\gamma x^{1 / \delta}\right)$ where $\gamma, \delta>0$. Then there exists a constant $C$ dependent on $\gamma, \delta$ such that uniformly on compacts of $(x, t)$

$$
s_{n}(\mathbb{H}(x, t))=O\left(\exp \left\{-C n^{\omega}\right\}\right), \quad n \rightarrow \infty,
$$

where $\omega=1$ if $0<\delta \leq 1$ and $\omega=1 / \delta$ if $\delta>1$.

Proof. As before whenever it leads to no confusion, we suppress the dependence on $(x, t)$ and assume $a=0$. By taking the co-analytic part of $\xi R$ and splitting $R$ by (7.22) one has

$$
\mathbb{H}(x, t)=\mathbb{H}\left(\Psi-\frac{c_{0} \xi\left(i \kappa_{0}\right)}{\kappa_{0}+i .}+\xi R_{0}\right),
$$

where $c_{0}$ is the norming constant for the bound stat $20-\kappa_{0}^{2}$ and $\left(y_{0}:=y(\cdot, 0)\right)$

$$
\Psi(k):=-\frac{1}{2 \pi i} \int_{\mathbb{R}+i h}(\cdot-k)^{-1} \xi\left(A_{0}-y_{0}^{-1} T_{0}\right) .
$$

\footnotetext{
${ }^{19}$ Much more specific statements regarding domains can be made.

${ }^{20}$ If there is no bound state then $c_{0}=0$.
} 
For $R_{0}$, the reflection coefficient from $q_{0}$, we use the representation [19]

$$
R_{0}(\lambda)=\frac{T_{0}(\lambda)}{2 i \lambda} \int_{0}^{\infty} e^{-2 i \lambda s} g(s) d s,
$$

where $g$ is some function for which we only need the bound

$$
|g(s)| \leq|q(s)|+\text { const } \int_{s}^{\infty}|q| .
$$

If $0<\delta \leq 1$ then the Fourier transform $G(\lambda):=\int_{0}^{\infty} e^{-2 i \lambda s} g(s) d s$ in (9.11) extends analytically to (at least) a strip and can be treated similarly to (9.10). In the case $\delta>1$ the part $\xi R_{0}$ of the symbol in (9.9) need not extend analytically from the real line. But it has a pseudo analytic extension. Due to our condition on $q$, the function $G(\lambda)$ admits (see, e.g. 68) a smooth bounded pseudo analytic continuation $G(\alpha, \beta)$ into $\mathbb{C}^{+}$with the property $(\delta>1)$

$$
|\bar{\partial} G(\alpha, \beta)| \lesssim K \exp \left\{-(Q / \beta)^{1 /(\delta-1)}\right\}, \quad K:=\int_{0}^{\infty} w|q|,
$$

where $Q$ is a constant dependent on $\gamma, \delta$. We are able now to evaluate the coanalytic part of $\xi R_{0}$ by the Green formula applied to the domain $\{0<\beta<h\}$ with any $h>\kappa_{0}$

$$
\begin{aligned}
\widetilde{\mathbb{P}}_{-}\left(\xi R_{0}\right) & =\frac{1}{2 \pi i} \int \frac{\xi(\lambda) R_{0}(\lambda)}{k-i 0-\lambda} d \lambda \\
& =\frac{1}{\pi} \int_{0<\beta<h} \frac{T_{0}(\lambda)}{2 i \lambda} \frac{\xi(\lambda) \bar{\partial} G(\alpha, \beta)}{\lambda-k} d \alpha d \beta \\
& +\frac{1}{2 \pi i} \int_{\mathbb{R}+i h} \frac{T_{0}(\lambda)}{2 i \lambda} \frac{\xi(\lambda) G(\alpha, \beta)}{k-\lambda} d \lambda \\
& +\frac{\omega}{\kappa_{0}+i k},
\end{aligned}
$$

where $\omega$ is an essential constant. We now insert (9.13) into (9.9). The term (9.15) can be combined with $\Psi$ to form a new one, $\Omega$. The term (9.16) joints in (9.9) the middle one to produce a partial fraction $\frac{\sigma}{\kappa_{0}+i k}$ which by Remark 9.5 can be neglected. Due to the rapid decay of $\xi(\lambda)$ along $\mathbb{R}+i h$, Proposition 9.4 applies and choosing $h=2 h_{0}$ we get

$$
s_{n}\left(\mathbb{H}\left(\Psi+\frac{\sigma}{\kappa_{0}+i \cdot}\right)\right)=O\left(e^{-h_{0} n}\right) .
$$

The term (9.14), which we denote by $\Lambda$, is therefore only one that needs some attention. By (9.7) and (9.12) we have

$$
\left|\xi_{x+i y, t}(\lambda) \bar{\partial} G(\alpha, \beta)\right| \lesssim K \xi_{x, t}(i \beta) \exp \left\{-(\sqrt{24 \beta t} \alpha)^{2}-(Q / \beta)^{1 /(\delta-1)}\right\} .
$$

Omitting straightforward but rather involved computations, we obtain

$$
\left\|\partial_{k}^{m} \Lambda\right\|_{\infty} \lesssim(m !)^{\delta} \widetilde{Q}^{-m}
$$

with some $\widetilde{Q}$ dependent on $\gamma$ and $\delta$. Theorems $9.1,9.2$ and Lemma 9.3 then yield

$$
s_{n}(\mathbb{H}(\Lambda))=O\left(\exp \left\{-C n^{1 / \delta}\right\}\right) .
$$

Combining (9.17) and (9.18) through (9.6) implies (9.8) with some smaller than in (9.18) constant $C$. 
Finally, the following theorem can be obtained by using techniques from this section (see 68, for more detail).

Theorem 9.7. Under conditions of Theorem 9.6 for any $t>0$ the operator-valued function $\partial_{t}^{m} \partial_{x}^{m} \mathbb{H}(x, t)$ (as an element of $\mathfrak{S}_{p}, 0<p \leq \infty$ ) is (1) entire if $0<\delta<2$, (2) analytic in the strip $|\operatorname{Im} x|<\frac{9 \sqrt{2}}{8} \gamma \sqrt{t}$ if $\delta=2$, and (3) in the Gevrey class $G^{\delta / 2}$ if $\delta>2$.

\section{The IST For The KDV EQUATION With STEP-LIKE INITIAL DATA}

In this section we finally state and prove our main result, which loosely speaking says: The problem (2.1)-(2.2) is well posed and its solution can be found by a suitable IST. With all the preparations done in the previous sections, the actual proof will be quite short.

Note that while the interest to well-posedness of integrable systems has been generated by the progress in soliton theory, well-posedness issues are typically approached by means of PDEs techniques [74] (norm estimates, etc.) and the IST is not usually employed. In soliton theory, in turn, well-posedness is commonly assumed (frequently even by default) and one applies the IST method to study the unique solution to (2.1)-(2.2) or any other integrable system. The paper [4] represents a rather rare example where the complete integrability of (2.1)-(2.2) with periodic initial data was used in a crucial way to prove some subtle well-posedness results for irregular $q$ which are not accessible by harmonic analysis means. In our case neither a priori well-posedness nor IST are readily available and we have to deal with both at the same time.

Solutions of the KdV can be understood in a number of different ways 74 (classical, strong, weak, etc.) resulting in a variety of different well-posedness results. Our definition is consistent with that in 47.

Definition 10.1 (Natural solution). Let $\left\{q_{n}(x, t)\right\}$ be a sequence of (classical) solutions of (2.1) with compactly supported initial data $q_{n}(x)$ converging in $L_{\mathrm{loc}}^{1}$ to $q(x)$. We call $q(x, t)$ a global natural solution to (2.1)-2.2) if

(1) for any $t>0$ uniformly on compacts of $\mathbb{R}$

$$
q(x, t)=\lim q_{n}(x, t), n \rightarrow \infty,
$$

independently of the choice of $q_{n}$.

(2) $q(x, t)$ is a classical solution of (2.1),

(3) $q(x, t)$ satisfies the initial condition (2.2) in the sense that

$$
q(x, t) \rightarrow q(x) \text { in } L_{\mathrm{loc}}^{1} \text { as } t \rightarrow+0 .
$$

Thus we understand well-posedness in a very strong sense. It also looks quite natural from the computational and physical point of view. Another feature of Definition 10.1 is that existence implies uniqueness and certain continuous dependence on the initial data.

Theorem 10.2 (Main Theorem). Assume that the initial data $q$ in (2.2) is subject to Hypothesis [2.1 with $w(x)=\exp \left(\gamma x^{1 / \delta}\right)$ where $\gamma, \delta>0$. Then the Cauchy problem (2.1)-(2.2) has a smooth global natural solution $q(x, t)$ (Definition 10.1) given by

$$
q(x, t)=-2 \partial_{x}^{2} \log \operatorname{det}(1+\mathbb{H}(x, t))
$$


where $\mathbb{H}(x, t)$ is the IST Hankel operator associated with $q$ (Definition 8.1). Singular numbers of $\mathbb{H}(x, t)$ decay uniformly on compacts of $(x, t)$ at the rate

$$
s_{n}(\mathbb{H}(x, t))=O\left(\exp \left\{-C n^{\omega}\right\}\right), \quad n \rightarrow \infty,
$$

where $\omega=\min \{1,1 / \delta\}$ and $C$ is a constant dependent on $\gamma, \delta$. Furthermore, for any $t>0$

(1) If $1<\delta<2$ then $q(x, t)$ is meromorphic on $\mathbb{C}$ with no poles on $\mathbb{R}$.

(2) If $\delta=2$ then $q(x, t)$ is meromorphic in the strip

$$
|\operatorname{Im} x|<\frac{9 \sqrt{2}}{8} \gamma \sqrt{t}
$$

with no poles on $\mathbb{R}$.

(3) If $\delta>2$ then $q(x, t)$ is in the Gevrey class $G^{\delta / 2}$.

Proof. Without loss of generality we can set the splitting point $a=0$. Since the problem (2.1)-(2.2) with initial data $\chi q$ is classical, one only needs to consider $q_{n}$ in Definition 10.1 such that $\chi q_{n}=\chi q$. The KdV equation with initial data $q_{n}(x)$ has a unique classical solution $q_{n}(x, t)$ computed by

$$
q_{n}(x, t)=-2 \partial_{x}^{2} \log \operatorname{det}\left(\mathbb{I}+\mathbb{H}_{n}(x, t)\right),
$$

where $\mathbb{H}_{n}(x, t)$ is the IST Hankel operator corresponding to $q_{n}$. By Theorem 9.7 $q_{n}(x, t)$ is a meromorphic function in $x$ on the entire complex plane. Consider the function $q(x, t)$ given by (10.2). By Theorem 9.7 it is well defined and at least Gevrey smooth. It remains to prove that $q(x, t)=\lim q_{n}(x, t), n \rightarrow \infty$, solves (2.1)-(2.2). By Theorems 8.2 and $9.7 q(x, t)$ is well defined and at least Gevrey smooth. We rewrite $q=q_{n}+\Delta q_{n}$, and insert this into (2.1):

$$
\begin{aligned}
& \partial_{t} q-6 q \partial_{x} q+\partial_{x}^{3} q \\
& =\partial_{t} \Delta q_{n}+3 \partial_{x}\left[\left(\Delta q_{n}-2 q\right) \Delta q_{n}\right]+\partial_{x}^{3} \Delta q_{n} .
\end{aligned}
$$

For $\Delta q_{n}$ we have (dropping subscript $\left.x, t\right)$

$$
\Delta q_{n}=-2 \partial_{x}^{2} \log \operatorname{det}\left(\mathbb{I}-(\mathbb{I}+\mathbb{H})^{-1}\left(\mathbb{H}-\mathbb{H}_{n}\right)\right) .
$$

It follows form (9.9) and (9.10) that for the symbol $\Delta \Psi_{n}$ of $\mathbb{H}-\mathbb{H}_{n}$ we have

$$
\Delta \Psi_{n}(k)=\frac{1}{2 \pi i} \int_{\mathbb{R}+i h} \frac{\xi(\lambda)\left(A_{0, n}(\lambda)-A_{0}(\lambda)\right) d \lambda}{\lambda-k} .
$$

But, as we know, $A_{0, n} \rightrightarrows A_{0}$ in $\mathbb{C}^{+}$as $n \rightarrow \infty$ and we can easily conclude that $\partial_{t}^{m} \partial_{x}^{l}\left(\mathbb{H}-\mathbb{H}_{n}\right)$ vanishes in the trace norm as $n \rightarrow \infty$. Therefore $\partial_{t}^{m} \partial_{x}^{l} \Delta q_{n} \rightarrow$ $0, n \rightarrow \infty$ and (10.5) immediately implies that $q(x, t)$ solves (2.1). (10.1) is proven in [69. The rest of the statement follows from Theorems 9.6 and 9.7

Theorem 10.2 concludes our study started in 70 of step like initial data with arbitrary behavior at $-\infty$. The relevance to Hankel operators was realized in [69] but we had to impose an additional condition to prove non-singularity of $\mathbb{I}+\mathbb{H}$. We conjectured in [69] that this condition can be removed. In [40, 41] we finally linked the IST and Hankel operators but were unable to completely remove that condition. The new condition was so weak and subtle that a counterexample would be extremely hard to construct. In [68] we conveniently used the language of Hankel operators to prove (1)-(3). The exact connection (10.3), based upon the AdamyanArov-Krein theory, between the decay of $q(x)$ at $+\infty$ and smoothness of $\mathbb{H}(x, t)$ 
(and hence $q(x, t))$ is first established here. Besides, in our previous papers we were more dependent on auxiliary results from other sources which come with somewhat stronger local conditions on $q(x)$. We also had a more complicated formula for the measure $\rho$ in the scattering data. The expression (7.20) is easiest possible. All this has resulted in a much more streamlined exposition.

\section{Some corollaries of the Main theOrem}

Theorem 10.2 readily implies a number of corollaries as well as quickly recovers and improves on many already known results. Below are some of them.

11.1. Hirota tau function. The explicit formula (10.2) immediately yields the representation

$$
\tau(x, t)=\operatorname{det}(\mathbb{I}+\mathbb{H}(x, t)),
$$

for the Hirota tau function [43, a well-known popular object of soliton theory. The substitution $q(x, t)=-2 \partial_{x}^{2} \tau(x, t)$ is commonly used as an ansatz to reduce the $\mathrm{KdV}$ equation to the so-called bilinear $\mathrm{KdV}$ which is advantageous in some situations. Formulas like (10.2) are particularly convenient for describing classes of exact solutions (see, e.g. [55]) and $\tau(x, t)$ typically appears as a Wronskian. We refer to [26], 61, 67, and [78 for (10.2) in the context of the Cauchy problem for the KdV. In our generality (10.2) is new.

11.2. Rate of convergence. The relation (10.3) means that the determinant in (10.2) rapidly converges. This fact, coupled with the recent progress in computing Fredholm determinants [8], suggests that, contrary to the common belief, (10.2) could be used for numerical evaluations.

11.3. Analyticity. Parts (1)-(3) of Theorem 10.2 say that any, no matter how rough, locally integrable initial profile $q(x)$ instantaneously evolves under the KdV flow into a smooth function $q(x, t)$. This effect, also called dispersive smoothing, has a long history. While being noticed long ago, its rigorous proof took quit a bit of effort even for box shaped initial data [60] (see also [80] for other integrable systems). Theorem 10.2 also implies that the rate of decay of $q(x)$ at $+\infty$ solely determines smoothness of $q(x, t)$ for any $q(x)$ essentially bounded from below.

More can be said if $0<\delta<2$. The solution $q(x, t)$ is then meromorphic in $x$ on the whole complex plane which means that $q(x, t)$ cannot vanish on a set of positive Lebesgue measure for any $t>0$ unless $q(x)$ is identically zero and we quickly recover and improve on many results of 779. E.g. assuming that $q(x)$ is absolutely continuous and short range, it is proven in [79] that $q(x, t)$ cannot have compact support at two different moments unless it vanishes identically. The techniques of [79] also rely on the IST and some Hardy space arguments.

If $\delta=2$ then part (2) of Theorem 10.2 says that $q(x, t)$ is meromorphic in a strip widening proportionally to $\sqrt{t}$. The closest known result [75] can only claim that $q(x, t)$ is real analytic and its proof requires strong decay at $-\infty$ as well as local $L^{2}$ integrability. The approach of 75 is based on the classical IST coupled with analysis of the Airy function and therefore quite involved. 


\section{WHAT WE DON'T KNOW BUT WOULD LIKE TO}

12.1. Slower decay at $+\infty$. The most important problem we are particularly concerned with is how much the decay condition at $+\infty$ could be relaxed. Due to the famous Bourgain result [11] the problem (2.1)-(2.2) is well-posed for $q \in L^{2}$. It is important to notice that the Bourgain's paper drew an enormous attention in the PDE/Harmonic analysis community to the well-posedness of (2.1)-(2.2) with singular data from Sobolev spaces with negative indices (see, e.g., 74 and the extensive literature cited therein). Thus developing IST techniques 21 for such initial data is arguably even more important and is a long overdue problem. It literally remains an uncharted territory. The formula (10.2) however cannot possibly hold as is for a number of reasons. We cautiously conjecture that a suitable IST can still be found if $q$ in Hypothesis 2.1 is merely square integrable at $+\infty$. At this point we are far from understanding how to deal with the mounting serious issues. In some particular (but interesting) cases the formulas for $R$ and $\rho$ in (7.13) and (7.20) are still well defined. The IST Hankel operator $\mathbb{H}(x, t)$ however need no longer be in the Sarason algebra 22 resulting in a lack of compactness. On the other hand the symbol of $\mathbb{H}(x, t)$ clearly says what the problems are and what can be tried to approach them. For instance, if $q(x)=O\left(1 / x^{2}\right),|x| \rightarrow \infty$, then $\mathbb{L}_{\chi_{a} q}$ may have infinite many negative bound states for any $a$ but the right reflection coefficient $R_{a}(k)$ off $q_{a}$ is well behaved for every $k \neq 0$ and at $k=0$ its argument may have a jump discontinuity of size $\gamma$ readily available from the asymptotic behavior of $q$. The infinite negative spectrum can be handled by applying an infinite chain of Darboux transforms [17. The jump discontinuity at zero can be modeled by the suitably defined analytic function $\left(\frac{k-i \varepsilon}{k+i \varepsilon}\right)^{\gamma / 2 \pi}$, with any $\varepsilon>0$, allowing us to "factor out" the undesirable behavior. This way (10.2) could be effectively regularized by singling out the behavior of $q(x, t)$ corresponding to the point $k=0$. Similarly, one can approach Wigner-von Neumann initial profiles $q(x) \sim A \frac{\sin 2 \omega x}{x},|x| \rightarrow \infty$. A new additional feature emerges 48 here. It is related to the so-called Wignervon Neumann resonance $\omega^{2}$ and says that the $\arg R(k)$ has a jump discontinuity at $\pm \omega$ of size $\gamma=2 \pi \omega|A|$. As in the previous example, the singular behavior of $R(k)$ is captured by suitably defined function $\left(b_{\mu}(k) b_{-\bar{\mu}}(k)\right)^{\gamma / 2 \pi}$, where $b_{\mu}$ is the Blaschke factor with zero $\mu=\omega+i \varepsilon$ for any $\varepsilon>0$. The formula (10.2) gains an extra term associated with the Wigner-von Neumann resonance. Same way a sum of different Wigner-von Neumann potentials can be handled. We have not worked out the details even for one resonance initial profile. But this would be particularly interesting as the Matveev's conjecture [58] on existence of bounded positon (breather) solutions could be then addressed. One of the challenges is that the spectral and scattering theory for long range potentials is not as well-developed as its short-range counterpart and there is no 'one stop shopping' like the seminal [19].

12.2. Asymptotic solutions. Much of the activity related to the Cauchy problem (2.1)-(2.2) is concerned with long time asymptotic behavior of its solution.

\footnotetext{
${ }^{21}$ Or at least understanding in what sense (2.1)-2.2 is more integrable than a generic PDE.

${ }^{22}$ Even boundedness of $\mathbb{H}(x, t)$ may in fact be lost.

${ }^{23}$ Which could under certain condition be an embedded positive bound state.
} 
The most powerful method to study this is arguably the nonlinear steepest descent method [18 based on the Riemann-Hilbert problem. A nice well-written exposition of this method for (2.1)-(2.2) is given in recent [42. Roughly speaking, this approach amounts to taking one (say right) basic scattering relation (6.8) and its complex conjugate and considering this pair as a two by two matrix Riemann-Hilbert problem for the row matrix $Y=\left(\begin{array}{ll}T y_{-} & y_{+}\end{array}\right)$. Asymptotics in each region of $(x, t)$ is then extracted from a very clever multi-step transformation (factorization, conjugation, contour deformation, etc.) of the original Riemann-Hilbert problem to the one that captures the asymptotic behavior in that particular region. No Hankel or Toeplitz operator explicitly appear this way but it is of course well-known [15] that the Riemann-Hilbert problem is essentially equivalent to invertibility of a certain Toeplitz operator ${ }^{24}$. It is therefore reasonable to ask if asymptotic solutions of (2.1) - (2.2) could be obtained entirely within the theory of Hankel/Toeplitz operators as effectively as using techniques of the Riemann-Hilbert problem? We don't have a clear vision if this could be the case. However translating the nonlinear steepest descent method into the language of Hankel would be of interest in its own right. We hope that more and deeper connections between soliton theory and the theory of Hankel operators could be uncovered this way.

It is of course one of our goals to analyze asymptotics of (2.1)-(2.2) with initial data subject to Hypothesis 2.1. A comprehensive treatment of the case $q(x) \rightarrow-h^{2}$ as $x \rightarrow-\infty$ in all asymptotic regions has been recently given in [23. We also refer to 23 for a extensive literature review. The main feature of this case is that such a step asymptotically splits into an infinite train of solitons twice as high as the step itsel 25. We cautiously conjecture that this type of behavior is universal as long as $\mathbb{L}_{q}$ has some negative essential spectrum. This also holds [50] if $q(x)$ tends to a periodic function at $-\infty$. It can be quite easily seen from the considerations of Subsection 9.2 that $q(x, t) \rightarrow 0$ uniformly in the region $x / t>h_{0}^{2}$ as $t \rightarrow \infty$. This is as much as we know at this point. We only mention that both, right and left, basic scattering relations are used in 23 to implement the nonlinear steepest descent method. In our case the left scattering relation need not exist.

12.3. Analyticity. Theorem 10.2 says that if $0<\delta<2$ then $q(x, t)$ is meromorphic on the whole complex plane for any $t>0$ and hence, as any meromorphic on $\mathbb{C}$ function, it is completely characterized by a countable number of time dependent parameters. Viewing a pure soliton solution as a meromorphic function of $x$ has a long story. In particular, the importance of pole dynamics was recognized by Kruskal 52 back in the early 70s for pure soliton solutions and has been actively studied since then (see also [2], 77, [16, 35] to mention just four). But very little is known about meromorphic solutions to the Cauchy problem (2.1)-(2.2). The problem boils down to the study of the meromorphic operator valued function $(\mathbb{I}+\mathbb{H}(x, t))^{-1}$. The general theory [73] only says that its poles depend continuously on $t$ and cannot appear or disappear. We are unaware of any relevant helpful results in the theory of Hankel operators.

12.4. Unification of the short-range and periodic ISTs. This is posed in [1] as an intriguing open problem. Why the periodic IST implies the short-range IST is

\footnotetext{
${ }^{24}$ As a matter of fact, Theorem 5.3 of principal importance to us, was originally found due to some problems having roots in the Riemann-Hilbert problem [36, 63.

${ }^{25}$ In nature this phenomenon can be seen in the so-called undular bore waves.
} 
explained in the classical book [61] (see also [77] and 26] for rigorous related results) but how the periodic IST emerges from the short range one does not appear to be well understood. We believe that to approach this problem one needs to consider (10.2) for a periodic function restricted to $(-\infty, a)$ and then let $a \rightarrow \infty$. We see two reasons why this approach should work: (1) the reflection coefficient off $(-\infty, a)$ is explicitly representable 64 in terms of spectral characteristics of the periodic problem; (2) the solution formula for the periodic case 61 has a similar to (10.2) structure with the tau function represented via the theta function. This suggests that the convergence should indeed take place. However we do not have any insight how difficult this problem could be. We cautiously suspect that the ideas and machinery used to find asymptotics of the so-called Toeplitz determinants [10] could be applied to our problem. If this indeed can be done within the theory of Hankel/Toeplitz operators then we might be able to avoid the complicated Riemann surfaces techniques commonly associated with the periodic IST.

\section{REFERENCES}

[1] Ablowitz, M.; Clarkson, P. Solitons, nonlinear evolution equations and inverse scattering. London Mathematical Society Lecture Note Series, 149. Cambridge University Press, Cambridge, 1991. xii+516 pp.

[2] Airault, H; McKean, H.; Moser, J. Rational and elliptic solutions of the Korteweg-de Vries equation and a related many-body problem, Commun. Pure Appl. Math. 30, (1977), 95-148.

[3] Aronszajn, N.; Donoghue, W. F. On exponential representations of analytic functions in the upper half-plane with positive imaginary part. J. Analyse Math. 5 (1957), 321-388.

[4] Atkinson, F. V. On the location of the Weyl circles. Proc. Roy. Soc. Edinburgh Sect. A 88 (1981), no. 3-4, 345-356.

[5] Aktosun, T. Inverse Schrödinger scattering on the line with partial knowledge of the potential. SIAM J. Appl. Math. 56 (1996), 219-31.

[6] Aktosun, T; Klaus, M.; van der Mee, C. On the Riemann-Hilbert problem for the one dimensional Schrödinger equation. J. Math. Phys. 34 (1993), 2651-90.

[7] Bona, Jerry L.; Weissler, Fred B. Pole dynamics of interacting solitons and blowup of complex-valued solutions of KdV. Nonlinearity 22 (2009), no. 2, 311-349.

[8] Bornemann, Folkmar. On the numerical evaluation of Fredholm determinants. Math. Comp. 79 (2010), no. 270, 871-915.

[9] Bötcher, A.; Grudsky S.; Spitkovsky, I. Toeplitz operators with frequency modulated semialmost periodic symbols. J. Fourier Anal. and Appl. 7 (2001), no. 5, 523-35.

[10] Bötcher, A.; Silbermann B. Analysis of Toeplitz operators. Springer-Verlag, Berlin, 2002. 665 pp.

[11] Bourgain, J. Fourier transform restriction phenomena for certain lattice subsets and applications to nonlinear evolution equations I, II. Geom. Funct. Anal., 3 (1993) :107-156, 209-262.

[12] Braun, M.; Sofianos, S.; Lipperheide, R. One-dimensional Marchenko inversion in the presence of bound states. Inverse Problems 11 (1995), L1-3.

[13] Carmona, R.; Lacroix, J. Spectral theory of random Schrödinger operators. Probability and its Applications. Birkhäuser Boston, Inc., Boston, MA, 1990. xxvi+587 pp.

[14] Cohen, Amy Solutions of the Korteweg-de Vries equation with steplike initial profile. Comm. Partial Differential Equations 9 (1984), no. 8, 751-806.

[15] Clancey, Kevin F.; Gohberg, Israel Factorization of matrix functions and singular integral operators. Operator Theory: Advances and Applications, 3. Birkhäuser Verlag, Basel-Boston, Mass., 1981. x+234 pp. ISBN: 3-7643-1297-1.

[16] Deconinck, Bernard; Segur, Harvey Pole dynamics for elliptic solutions of the Korteweg-de Vries equation. Math. Phys. Anal. Geom. 3 (2000), no. 1, 49-74

[17] Degasperis, A.; Shabat, A. Construction of reflectionless potentials with infinite discrete spectrum. Teoret. Mat. Fiz. 100, no. 2 (1994) , 230-247.

[18] Deift, P.; Zhou, X. A steepest descent method for oscillatory Riemann-Hilbert problems. Asymptotics for the MKdV equation. Ann. of Math. (2) 137 (1993), no. 2, 295-368. 
[19] Deift, P.; Trubowitz, E. Inverse scattering on the line. Comm. Pure Appl. Math. 32 (1979), no. $2,121-251$.

[20] Dybin, V.; Grudsky S. Introduction to the theory of Toeplitz operators with infinite index. Birkhäuser Verlag, Basel, 2002. xii+299 pp.

[21] Dyn'kin, E. M. Pseudoanalytic continuation of smooth functions. Uniform scale. In Mathematical programming and related questions (Proc. Seventh Winter School, Drogobych, 1974), Theory of functions and functional analysis (Russian), pages 40-73. Central Ekonom.-Mat. Inst. Akad. Nauk SSSR, Moscow, 1976, English translation in Amer. Math. Soc. Transl. (2), Vol. 115 (1980), 33-58.

[22] Dyson F. J., Fredholm determinants and inverse scattering problems, Comm. Math. Phys., 1976, 47(2), 171-183.

[23] Egorova, I.; Gladka, Z.; Kotlyarov, V.; Teschl, G. Long-time asymptotics for the Korteweg-de Vries equation with step-like initial data. Nonlinearity, 26 (2013), 7, 1839-1864.

[24] Egorova, Iryna; Grunert, Katrin; Teschl, Gerald On the Cauchy problem for the Korteweg-de Vries equation with steplike finite-gap initial data. I. Schwartz-type perturbations. Nonlinearity 22 (2009), no. 6, 1431-1457.

[25] Egorova, Iryna; Teschl, Gerald On the Cauchy Problem for the Korteweg-de Vries Equation with Steplike Finite-Gap Initial Data II. Perturbations with Finite Moments, J. Anal. Math. 115 (2011), 71-101.

[26] Ercolani, N.; McKean, H. P. Geometry of KdV. IV. Abel sums, Jacobi variety, and theta function in the scattering case. Invent. Math. 99 (1990), no. 3, 483-544.

[27] Fokas, A. S.; Lenells, J. The unified method: I. Nonlinearizable problems on the half-line. J. Phys. A 45 (2012), no. 19, 195201, 38 pp.

[28] Fokas, A. S. Integrable nonlinear evolution equations on the half-line. Comm. Math. Phys. 230 (2002), no. 1, 1-39.

[29] Garnett, John B. Bounded analytic functions. Revised first edition. Graduate Texts in Mathematics, 236. Springer, New York, 2007. xiv+459 pp.

[30] Gérard, Patrick; Grellier, Sandrine Invariant tori for the cubic Szegö equation. Invent. Math. 187 (2012), no. 3, 707-754.

[31] Gérard, Patrick; Grellier, Sandrine The cubic Szegő equation. Ann. Sci. Éc. Norm. Supér. (4) 43 (2010), no. 5, 761-810.

[32] Gesztesy, F.; Karwowski, W.; Zhao, Z. Limits of soliton solutions. Duke Math. J. 68 (1992), no. $1,101-150$.

[33] Gesztesy, F.; Nowell, R.; Pötz, E. One-dimensional scattering theory for quantum systems with nontrivial spatial asymptotics. Differential Integral Equations 10 (1997), no. 3, 521-546.

[34] Gesztesy, F.; Simon, B. Inverse spectral analysis with partial information on the potential. I. The case of an a.c. component in the spectrum. Papers honouring the 60th birthday of Klaus Hepp and of Walter Hunziker, Part II (Zürich, 1995), Helv. Phys. Acta 70 (1997), no. $1-2,66-71$.

[35] Gesztesy, Fritz; Unterkofler, Karl; Weikard, Rudi An explicit characterization of CalogeroMoser systems. Trans. Amer. Math. Soc. 358 (2006), no. 2, 603-656

[36] Govorov, N. V. Riemann's boundary problem with infinite index. Edited and with an introduction and an appendix by I. V. Ostrovskiu. Translated from the 1986 Russian original by Yu. I. Lyubarskiî. Operator Theory: Advances and Applications, 67. Birkhäuser Verlag, Basel, 1994. xii+252 pp. ISBN: 3-7643-2999-8.

[37] Grébert, B.; Weder, R. Reconstruction of a potential on the line that is a priori known on the half line. SIAM J. Appl. Math. 55 (1995), 242-54.

[38] Grudsky, S.M. Toeplitz operators and the modelling of oscillating discontinuities with the help of Blaschke products. Operator theory: Advances and Applications, v. 121, Birkhäuser Verlag, Basel, 2001, pp. 162-193.

[39] Grudsky, Sergei; Shargorodsky, Eugene. Applications of Blaschke products to the spectral theory of Toeplitz operators. Blaschke Products and Their Applications, Fields Institute Communications Volume 65, 2013, 1-30 pp.

[40] Grudsky, S; Rybkin, A. On positive type initial profiles for the KdV equation. to appear in Proc Amer Math Soc.

[41] Grudsky, S; Rybkin, A. On Toeplitz and Hankel Operators with Oscillatory Symbols Containing Blaschke Products and Applications to the KdV Equation. Operator Theory: Advances and Applications, Vol. 228 (2013), 127-150. 
[42] Grunert, Katrin; Teschl, Gerald Long-time asymptotics for the Korteweg-de Vries equation via nonlinear steepest descent. Math. Phys. Anal. Geom. 12 (2009), no. 3, 287-324.

[43] Hirota, R. Exact solution of the Korteweg de Vries equation for multiple collisions of solitons. Phys. Rev. Lett. 27 (1971), 1192-1194.

[44] Hruslov, E. . Ja. Asymptotic behavior of the solution of the Cauchy problem for the Kortewegde Vries equation with steplike initial data. Mat. Sb. (N.S.) 99(141) (1976), no. 2, 261-281, 296.

[45] Its, Alexander R. The Riemann-Hilbert problem and integrable systems. Notices Amer. Math. Soc. 50 (2003), no. 11, 1389-140.

[46] Nikolski, N. K. Operators, functions, and systems: An easy reading. Volume 1: Hardy, Hankel and Toeplitz. Mathematical Surveys and Monographs, vol. 92, Amer. Math. Soc., Providence, 2002. 461 pp.

[47] Kappeler, T.; Topalov, P. Global wellposedness of $K d V$ in $H^{-1}(\mathbb{T}, \mathbb{R})$. Duke Math. J. Volume 135, Number 2 (2006), 327-36.

[48] Klaus, Martin (1991) Asymptotic behavior of Jost functions near resonance points for Wigner-von Neumann type potentials. J. Math. Phys. 32, no. 1, 163-174.

[49] Krichever, I.; Novikov, S. P. Periodic and almost-periodic potentials in inverse problems. Inverse Problems 15 (1999), no. 6, R117-R144.

[50] Khruslov, E. Ya.; Kotlyarov, V. P. Soliton asymptotics of nondecreasing solutions of nonlinear completely integrable evolution equations. Spectral operator theory and related topics, 129-180, Adv. Soviet Math., 19, Amer. Math. Soc., Providence, RI, 1994

[51] Kotani, S.; Ushiroya, N. One-dimensional Schrödinger operators with random decaying potentials. Comm. Math. Phys. 115 (1988), no. 2, 247-266.

[52] Kruskal, Martin D. The Korteweg-de Vries equation and related evolution equations. Nonlinear wave motion (Proc. AMS-SIAM Summer Sem., Clarkson Coll. Tech., Potsdam, N.Y., 1972), pp. 61-83. Lectures in Appl. Math., Vol. 15, Amer. Math. Soc., Providence, R.I., 1974.

[53] Lenells, J.; Fokas, A. S. The unified method: III. Nonlinearizable problems on the interval. J. Phys. A 45 (2012), no. 19, 195203, 21 pp.

[54] Lenells, J.; Fokas, A. S. The unified method: II. NLS on the half-line with t-periodic boundary conditions. J. Phys. A 45 (2012), no. 19, 195202, 36 pp.

[55] Ma, Wen-Xiu; You, Yuncheng Solving the Korteweg-de Vries equation by its bilinear form: Wronskian solutions. Trans. Amer. Math. Soc. 357 (2005), no. 5, 1753-1778.

[56] Marchenko, V. A. The Cauchy problem for the KdV equation with nondecreasing initial data. What is integrability?, 273-318, Springer Ser. Nonlinear Dynam., Springer, Berlin, 1991.

[57] Marchenko, V.A. Sturm-Liouville operators and applications. Translated from the Russian by A. Iacob. Operator Theory: Advances and Applications, 22. Birkh $\backslash\{$ a $\}$ user Verlag, Basel, 1986. xii+367 pp.

[58] Matveev, V. Positons: slowly decreasing analogues of solitons. (Russian) Teoret. Mat. Fiz. 131 (2002), no. 1, 44-61; translation in Theoret. and Math. Phys. 131 (2002), no. 1, 483-497.

[59] McLeod, J. B.; Olver, P. J. The connection between partial differential equations soluble by inverse scattering and ordinary differential equations of Painlevé type. SIAM J. Math. Anal. 14 (1983), no. 3, 488-506.

[60] Murray, Amy Cohen Solutions of the Korteweg-de Vries equation from irregular data. Duke Math. J. 45 (1978), no. 1, 149-181.

[61] Novikov, S.; Manakov, S. V.; Pitaevskiı̌, L. P.; Zakharov, V. E Theory of solitons. The inverse scattering method. Translated from the Russian. Contemporary Soviet Mathematics. Consultants Bureau [Plenum], New York, 1984. xi+276 pp. ISBN: 0-306-10977-8

[62] Novikov, S. P. A periodic problem for the Korteweg-de Vries equation. I. (Russian) Funkcional. Anal. i Priložen. 8 (1974), no. 3, 54-66. English translation: Functional Anal. Appl. 8 (1974), no. 3, 236-246 (1975).

[63] Ostrovskiŭ, I. V. Solvability conditions for the homogeneous Riemann boundary problem with an infinite index. Entire and subharmonic functions, 107-135, Adv. Soviet Math., 11, Amer. Math. Soc., Providence, RI, 1992.

[64] Pavlov, B. S.; Smirnov, N. V. Resonance scattering by a one-dimensional crystal and a thin film. Vestnik Leningrad. Univ. 10 (1982), 307-318.

[65] Pearson, D. B. Singular continuous measures in scattering theory. Comm. Math. Phys. 60 (1978), no. 1, 13-36. 
[66] Peller, Vladimir V. Hankel operators and their applications. Springer Monographs in Mathematics. Springer-Verlag, New York, 2003. xvi+784 pp. ISBN: 0-387-95548-8.

[67] Pöppe, Ch., The Fredholm determinant method for the KdV equations, Phys. D, 1984, 13(12), $137-160$.

[68] Rybkin, Alexei. Spatial analyticity of solutions to integrable systems. I. The KdVcase Communications in Partial Differential Equations, Volume 38 (2013), Issue 5, 802-822.

[69] Rybkin, A. The Hirota $\tau$-function and well-posedness of the KdV equation with an arbitrary step like initial profile decaying on the right half line, Nonlinearity 24 (2011), 2953-2990.

[70] Rybkin, Alexei Meromorphic solutions to the KdV equation with non-decaying initial data supported on a left half line. Nonlinearity 23 (2010), no. 5, 1143-1167.

[71] Rundell, W.; Sacks P. On the determination of potential without bound state data. J. Comput. Appl. Math. 55 (1994), 325-47.

[72] Simon, Barry Trace ideals and their applications. Second edition. Mathematical Surveys and Monographs, 120. American Mathematical Society, Providence, RI, 2005. viii+150 pp. ISBN: 0-8218-3581-5.

[73] Steinberg, S. Meromorphic families of compact operators. Arch. Ration. Mech. Anal. 31 (1968/19693) 72-9.

[74] Tao, Terence Nonlinear dispersive equations. Local and global analysis. CBMS Regional Conference Series in Mathematics, 106. Published for the Conference Board of the Mathematical Sciences, Washington, DC; by the American Mathematical Society, Providence, RI, 2006. xvi+373 pp. ISBN: 0-8218-4143-2.

[75] Tarama, S. Analyticity of solutions of the Korteweg-de Vries equation. J. Math. Kyoto Univ. 44 (2004), no. 1, 1-32.

[76] Teschl, Gerald Mathematical methods in quantum mechanics. With applications to Schrödinger operators. Graduate Studies in Mathematics, 99. American Mathematical Society, Providence, RI, 2009. xiv+305 pp.

[77] Venakides, S. The infinite period limit of the inverse formalism for periodic potentials. Comm. Pure Appl. Math. 41 (1988), no. 1, 3-17.

[78] Venakides, S. Long time asymptotics of the Korteweg - de Vries equation. Trans. Amer. Math. Soc. 293 (1986), no. 1, 411-419.

[79] Zhang, Bing Yu Unique continuation for the Korteweg-de Vries equation. SIAM J. Math. Anal. 23 (1992), no. 1, 55-71.

[80] Zhou, Xin. Strong regularizing effect of integrable systems. Comm. Partial Differential Equations 22 (1997), no. 3-4, 503-526.

Departamento de Matematicas, CinVestaV del I.P.N. Aportado Postal 14-740, 07000 Mexico, D.F., Mexico.

E-mail address: grudsky@math.cinvestav.mx.

Department of Mathematics and Statistics, University of Alaska Fairbanks, PO Box 756660, FAIRBANKS, AK 99775

E-mail address: arybkin@alaska.edu 